\title{
Generation of Anatomically Inspired Human Airway Tree Using Electrical Impedance Tomography: A Method to Estimate Regional Lung Filling Characteristics
}

\author{
Majid Zamani, Member, IEEE, Merja Kallio, Richard Bayford, Life Senior Member, IEEE, and \\ Andreas Demosthenous, Fellow, IEEE
}

\begin{abstract}
The purpose of lung recruitment is to improve and optimize the air exchange flow in the lungs by adjusting the respiratory settings during mechanical ventilation. Electrical impedance tomography (EIT) is a monitoring tool that allows to measure regional pulmonary filling characteristics or filling index (FI) during ventilation. The conventional EIT system has limitations which compromise the accuracy of the FI. This paper proposes a novel and automated methodology for accurate FI estimation based on EIT images of recruitable regional collapse and hyperdistension during incremental positive end-expiratory pressure. It identifies details of the airway tree (AT) to generate a correction factor to the FIs providing an accurate measurement. Multiscale image enhancement followed by identification of the AT skeleton with a robust and self-exploratory tracing algorithm is used to automatically estimate the FI. AT tracing was validated using phantom data on a ground-truth lung. Based on generated phantom EIT images, including an established reference, the proposed method results in more accurate FI estimation of $65 \%$ in all quadrants compared with the current state-of-the-art. Measured regional filling characteristics were also examined by comparing regional and global impedance variations in clinically recorded data from ten different subjects. Clinical tests on filling characteristics based on extraction of the AT from the resolution enhanced EIT images indicated a more accurate result compared with the standard EIT images.
\end{abstract}

Index Terms - Adaptive resolution enhancement, airway tree morphology, circular quantizer, distortion embedding, electrical impedance tomography (EIT), lung filling mechanics, optimal airway tree skeleton, self-exploratory tracing algorithm.

\section{INTRODUCTION}

$\mathrm{E}_{\mathrm{c}}^{\mathrm{c}}$ LECTRICAL impedance tomography (EIT) can provide continuous, real-time and non-invasive imaging by measuring the internal impedance related to physiological change in the human body from a series of surface electrodes placed on it [1]. In recent years there has been significant interest in monitoring regional lung changes using EIT, especially in designing patient-specific ventilation management leading to reduced incidence of ventilator-induced lung injury [2]. It has been shown that cyclic malfunctioning of collapsed and overdistended regions can lead to an increase in

Manuscript received 25, February 2021; revised September 22, 2021 and November 22, 2021. This work was supported in part by the Engineering and Physical Sciences Research Council (EPSRC) under grant EP/T001259/1, and in part by the European Commission under agreement no. 668259 (http://cradlproject.org).

M. Zamani and A. Demosthenous are with the Department of Electronic and Electrical Engineering, University College London, Torrington Place, London WC1E7JE, UK. (e-mail: m.zamani@ucl.ac.uk, a.demosthenous@ucl.ac.uk). inflammatory cytokines [3], thus the lung itself is an underlying cause in the multiple-organ failure sequence [4].

An important indicator used to assess the state of the lung physiology is the filling index (FI) which indicates whether a lung region fills at an increased or decreased rate compared with other regions. This assists the ventilation procedures for prevention of alveolar collapse and regional overdistension. The FI is calculated from tracings of regional tidal volumes compared with global tidal volumes measured with EIT images. The calculation of FI was the focus of several papers [5]-[10]. In [5], [6], the FI parameter was examined by first plotting the impedance change of the region of interest, for example, the right anterior (RA) against the global signal to form a curve. The curve then was fitted using the Levenberg-Marquardt method with the following equation:

$$
I(g)=\kappa \cdot g^{\mathrm{FI}}+\delta
$$

where $I(g)$ is the impedance, FI is the filling index in a region of interest, $\kappa$ and $\delta$ are constants. FI $<1$ indicates a relatively increased rate and FI $>1$ a relatively decreased rate, which could indicate recruitment. FI $=1$ indicates the lung region behaved the same as the global signal. In [7]-[10], the seconddegree polynomial function $y=a x^{2}+b x+c$ was used to fit the extracted regional-global curve with ' $a$ ' the second-degree polynomial coefficient. ' $a$ ' characterizes the curve linearity of the plot and describes the lung filling characteristics of the selected region. $a \sim 0$ indicates regional tidal volume change, which occurs during the whole inspiration homogeneously. $a>$ 0 indicates initial low regional tidal volume change compared with the average filling characteristics. This indicates possible regional collapse suggesting lung volume recruitment of the region. $a<0$ indicates late low regional tidal volume change compared with the average filling characteristics, which might occur during hyperdistention of the region.

The methods in [5]-[10] employ low or ultra-low resolution EIT images as a reflection of the airway tree (AT) functionality in the desired lung regions which limits the accuracy of the FI parameter. For example, the FI is calculated from its relevant impedance $\left(Z_{\mathrm{RA}}\right)$ compared with the global impedance. $Z_{\mathrm{RA}}$ is the integral of impedance values in the right anterior (RA)

M. Kallio is with PEDEGO Research Unit, Medical Research Center, University of Oulu and Department of Children and Adolescents, Oulu University Hospital, Oulu, Finland (e-mail: merja.kallio@oulu.fi).

R. Bayford is with the Department of Natural Sciences, Middlesex University, The Burroughs, London NW4 6BT, U.K. (e-mail: r.bayford@mdx.ac.uk). 


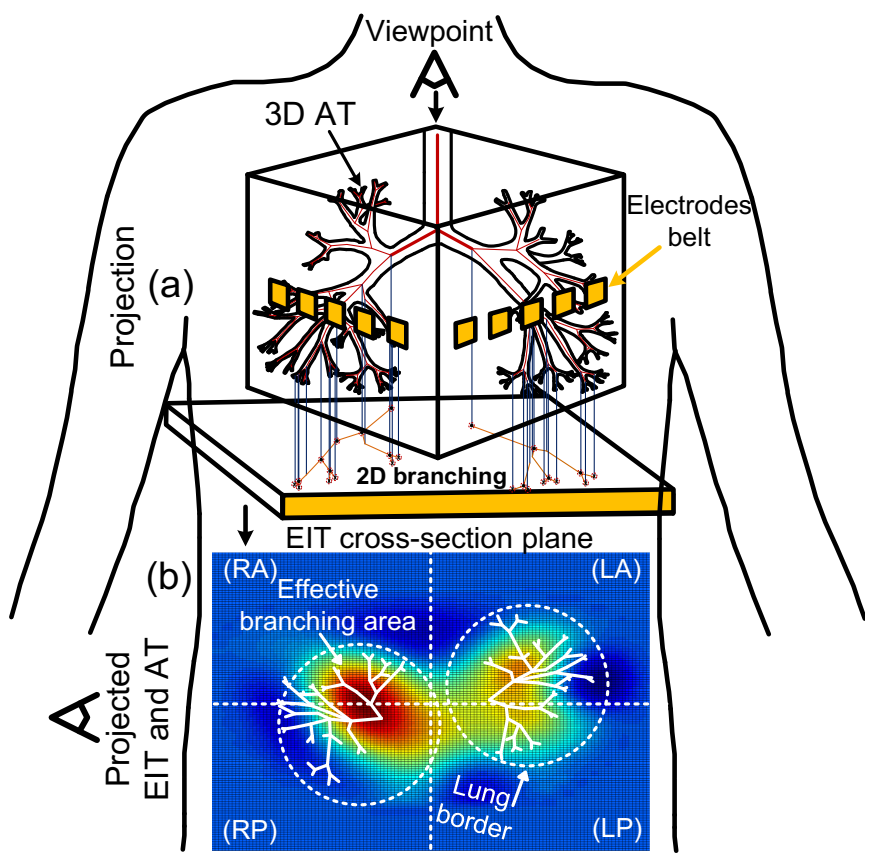

Fig. 1. (a) Projection of a 3D anatomical-like AT on the EIT cross-sectional plane from reference viewpoint. (b) The projected 2D AT is aligned with captured regional impedance variations in EIT image. The volume of lung in the EIT image is recorded as resistance distribution displayed in a matrix $\left(Z_{\text {matrix }}\right)$ of dimension $32 \times 32$. Regions identified in the lung: right anterior (RA), left anterior (LA), right posterior (RP) and left posterior (LP).

region and $E_{\mathrm{RA}}$ is the integral error due to lack of spatial (or spatial and temporal [11]) resolution on the impedance matrix $Z_{\text {matrix }}$ whose initial dimensions are $32 \times 32$. The calculated regional impedance of $\mathrm{RA}\left(Z_{\mathrm{RAE}}\right)$ is expressed as:

$$
Z_{\mathrm{RAE}}=\sum_{i=16}^{32} \sum_{j=1}^{16} Z_{\text {matrix }}=Z_{\mathrm{RA}}+E_{\mathrm{RA}} \text {. }
$$

$Z_{\mathrm{RAE}}$ is then used to extract a curve and fitting coefficients. $E_{\mathrm{RA}}$ in (2) embeds higher and lower slopes to the error-free $Z_{\mathrm{RAE}} / Z_{\mathrm{GL}}$ graph, where $Z_{\mathrm{GL}}$ is the global integral impedance of $Z_{\text {matrix }}\left(Z_{\mathrm{GL}}=\sum_{i=1}^{32} \sum_{j=1}^{32} Z_{\text {matrix }}+E_{\mathrm{GL}}\right)$. Therefore, the FI coefficient is derived based on rough estimation of fitting coefficients [5]-[10] on an error-embedded graph without identifying the spatial error.

This paper proposes image partial pixel sampling for accurate estimation of recruitable alveolar collapse and hyperdistention, thus providing an accurate FI calculation compared with [5]-[10]. The proposed approach decorrelates and minimizes $E_{\mathrm{RA}}$ by detailed extraction of the global-regional AT model of the lungs to estimate the filling characteristics based on the concept shown in Fig. 1.

When EIT boundary data is measured with a single plane of electrodes it provides a two-dimensional (2D) impedance map which includes the lung's volumetric functionality above and below the plane defined by the electrode belt. The threedimensional (3D) AT of the lungs is buried in a 2D EIT crosssectional plane shown in Fig. 1(b). The reference viewpoint is the node shown on top of the AT in Fig. 1(a) used to project the AT to the EIT plane. The EIT image is processed through upscaling and spatial enhancement to accurately project the $2 \mathrm{D}$ trajectories of the AT dichotomous branching in the cross-

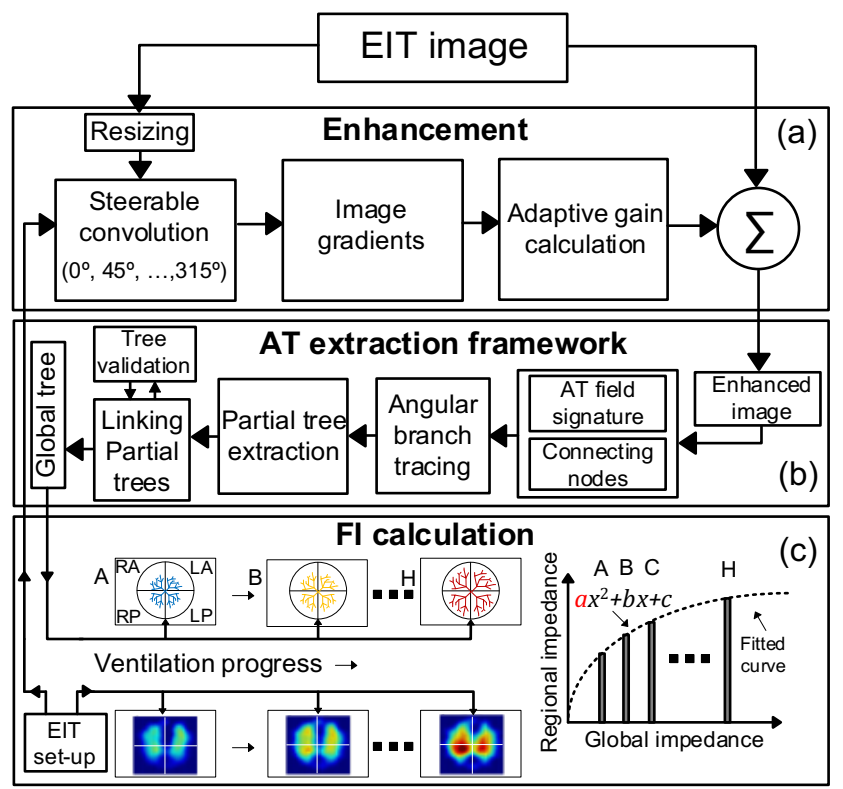

Fig. 2. Functional diagram of the proposed algorithm for FI calculation. (a) Pre-processing for EIT resolution enhancement, (b) AT extraction, and (c) fitting of filling graph and estimating fitting coefficient $a$.

section plane. The extracted 2D AT provides an average nonuniform correction coefficient (ANCC) that reduces the errors in regional FI characteristics of the lungs. Hence, the corrected impedance integral in the RA quadrant $\left(Z_{\mathrm{RAC}}\right)$ is:

$$
Z_{\mathrm{RAC}}=\mathrm{ANCC} \cdot Z_{\mathrm{RAE}} \quad 0<\mathrm{ANCC}<1 .
$$

The rest of the paper is organized as follows. Section II outlines the FI calculation method and Section III describes the set-up used to validate the AT tracing using a ground-truth lung. Section IV details the results of AT tracing and calculated FIs. Concluding remarks are drawn in Section V.

\section{FiLLING Index CALCULATION}

The operation of the proposed FI calculation method is summarized in Fig. 2. There are three steps: 1) Resolution enhancement to provide higher quality EIT images; 2) Recognition and extraction of a 2D regional-global AT; and 3) FI estimation for each quadrant [e.g. RA in Fig. 1(b)] of the lungs.

1) Resolution enhancement: Because features are barely distinguishable in the original EIT images, a multi-scale spatial resolution enhancement technique (exploiting distortion embedding) is used to better distinguish low level or low contrast features. The resolution enhancement consists of three processing steps [Fig. 2(a)]. First, the image gradients are computed by convolving the steered first four derivative operators utilizing Gaussian basis functions. Second, the highest convolution response from eight orientations $\left(0^{\circ}, 45^{\circ}\right.$, $90^{\circ}, 135^{\circ}, 180^{\circ}, 225^{\circ}, 270^{\circ}$ and $315^{\circ}$ ) is identified. Third, the important features of the original image are enhanced by embedding the adaptively weighted orientations with the highest convolution response.

2) AT extraction: Fig. 2(b) shows the schematic flow of the AT extraction algorithm. It is an automated self-exploratory tracing algorithm which extracts the AT structure from the 


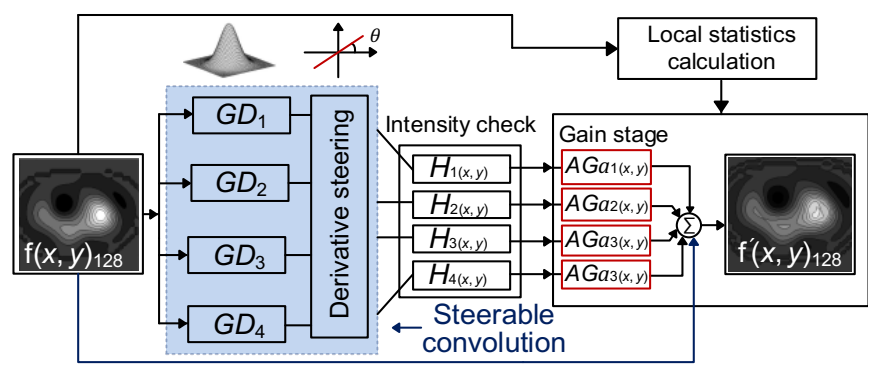

Fig. 3. Block diagram of the resolution enhancement method. $H_{k\{1, \ldots 4\}}(x, y)$ are the gradients of the steerable convolution with low resolution image, $\mathrm{f}(x, y)_{128}$ in this example. The gradients $H_{k\{1, \ldots 4\}}(x, y)$ are checked for highest intensity and fed to the gain stage. The gain stage $A G a_{k\{1, \ldots, 4\}}(x, y)$ adaptively tunes the regional resolution to highlight the hidden variations.

resolution enhanced EIT images. First, the preliminary information including connecting nodes and airflow field are computed (see Section III.B) and applied to the main processing core for AT tracing. Second, the AT segment centerlines are traced using a circular quantizer. The main AT segments are traced together with their diameter guided by the centerlines to realize an anatomical-like structure in the AT model. Third, all the identified AT segments are connected to form a composite cross-sectional view of the AT pattern in the lungs.

3) FI calculation: Fig. 2(c) shows the FI calculation in the ventilation phase. The filling characteristics are computed by comparing the integral impedance of pixels indicating AT with the global impedance $Z_{\mathrm{GL}}$ of the lungs $\left(Z_{\mathrm{GL}}=Z_{\mathrm{RA}}+Z_{\mathrm{LA}}+\right.$ $Z_{\mathrm{RP}}+Z_{\mathrm{LP}}$ ) obtained from EIT images. Determining the number of AT pixels provides the value of ANCC and results in an accurate FI estimation.

\section{EXTRACTION OF THE AIRWAY TREE}

\section{A. Adaptive Image Resolution Enhancement Using Multi-Scale Distortion Embedding}

Resolution enhancement (or resolution embedding) is a technique used in different fields, but especially in image processing. It uses advanced statistical pattern analysis [12], [13] and deep learning on the processing side [14] to embed more spatial accuracy into an image. The method described in [15] is used in this study for image spatial resolution enhancement. It captures and projects the regional variations in both scale and orientation, and superimposes adaptively the weighted features with coarse-fine scales into the original image:

$$
\mathrm{f}^{\prime}(x, y)=\mathrm{f}(x, y)+\sum_{k=1}^{n} A G a_{k}(x, y) H_{k}(x, y)
$$

where $\mathrm{f}^{\prime}(x, y)$ is the enhanced image, $n$ is the number of kernels used in the steerable convolution, and $A G a_{k}(x, y)$ is the signaladaptive gain for the $k^{\text {th }}$ image convolution. $H_{k}(x, y)$ is the gradient image obtained by convolving the EIT image $\mathrm{f}(x, y)$ with the $k^{\text {th }}$ kernel. First, the low resolution $\mathrm{f}(x, y)$ image is upscaled using bicubic interpolation to the desired size (e.g. to $128 \times 128$, denoted as $\mathrm{f}(x, y)_{128}$ in Fig. 3.) The Gaussian kernel derivatives $\left(G D_{1} \ldots G D_{4}\right)$ form a steerable filter dictionary $G D_{k\{1, \ldots 4\}}(\sigma, \theta)$ at an arbitrary standard-deviation $\sigma$ and angle $\theta$ [16], so they precisely capture the non-stationary localized variations in sub-bands representing different
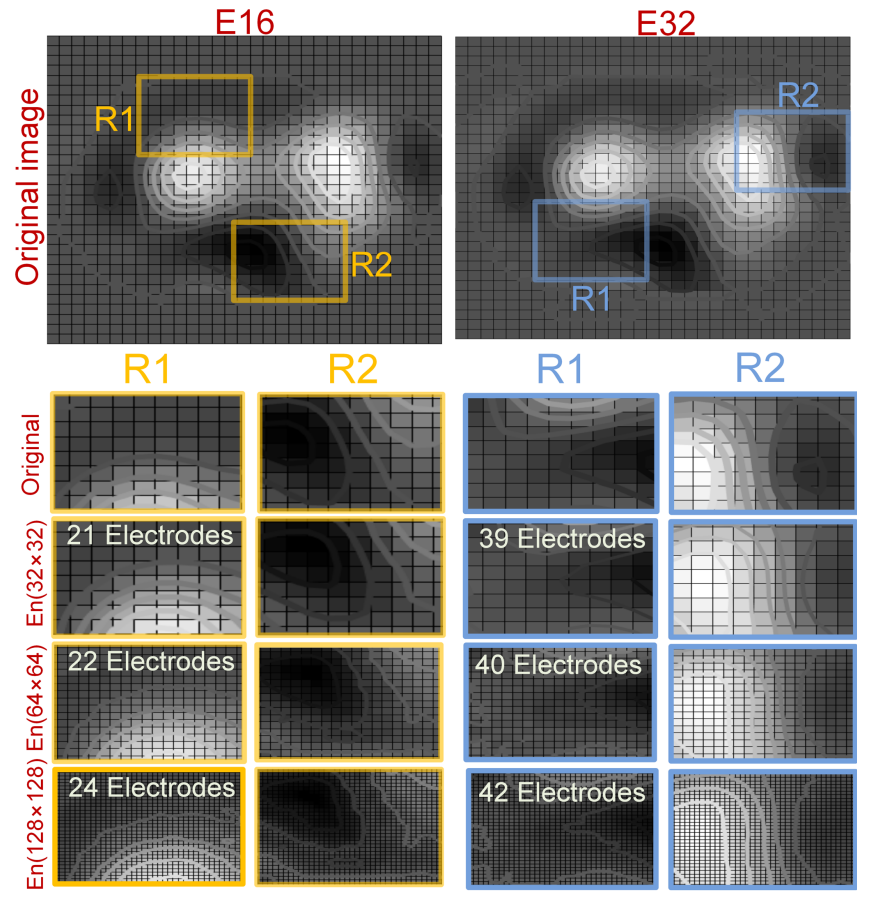

Fig. 4. The columns are EIT images from different electrode recording configurations [16 electrodes (E16) and 32 electrodes (E32)] [15]. Areas R1 and R2 have been selected on edges and curvatures to illustrate enhancement intensity distribution. The first row shows the partially sampled original images in regions R1 and R2. The second row shows the resolution enhanced EIT images En $(32 \times 32)$ without resizing. The third and the fourth rows show the effect of resolution enhancement on the resized EIT images $\operatorname{En}(64 \times 64)$ and $\operatorname{En}(128 \times 128)$. Contour plots are superimposed to make the regional variations clearer. Finer resolution is observed as the grid size varies in each column $\{\operatorname{En}(32 \times 32) \rightarrow \operatorname{En}(64 \times 64) \rightarrow \operatorname{En}(128 \times 128)\}$ as a result of embedding high-resolution features in smaller image patches. Each row also shows the equivalent number of electrodes; for example, En $(64 \times 64)$ represents a recording set-up with 22 electrodes. The number of electrodes is quantified based on the metric proposed in [15].

decomposition scales. The image derivatives $H_{k\{1, \ldots .4\}}(x, y)$ are obtained by convolving the resized image $\mathrm{f}(x, y)_{128}$ with the steered Gaussian derivative kernels $G D_{k\{1, \ldots 4\}}(\sigma, \theta)$ as shown in Fig. 3. Deep decomposition and enhancement are accomplished by setting $\sigma=0.7$ and the filter kernel size to $3 \times 3$ pixels. Having decomposed the original EIT image into sub-bands, a gain transfer function is created to adaptively escalate the regional variations at each specific sub-band. For each pixel $(x, y)$ the highest filter response is retained for adaptive gain adjustment. The regional quantization model (RQM) proposed in [15] employs pixel-wise adaptive gain $A_{G a} a_{k\{1, \ldots, 4\}}(x, y)$ tuning to generate the optimal level of adjustment in each projected image after convolution. RQM assigns more resolution to those directional edges which have higher standard deviation and embeds high-order curvatures into the EIT images while suppressing noise. The final step superimposes the modified sub-bands in all regions of the original image $\mathrm{f}(x, y)$ to enhance the spatial resolution. This process is shown in Fig. 4. It highlights areas of color-coded rectangles for visual assessment of the resolution enhancement. Using RQM it is shown in [15] that for belts containing 16 electrodes with noise-free recording of an upscaled $128 \times 128$ 


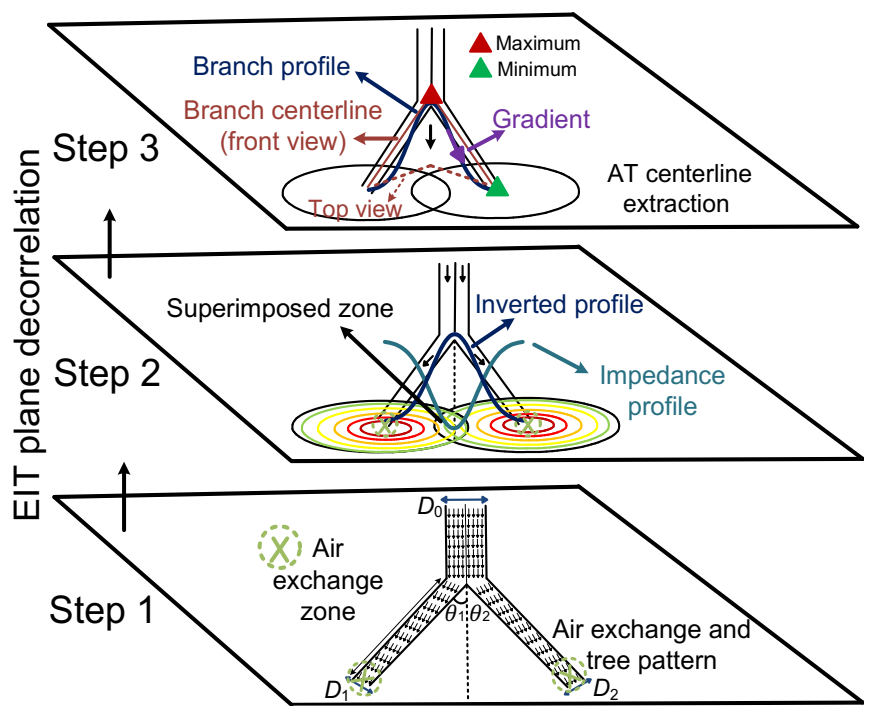

Fig. 5. EIT plane decorrelation steps. Step 1 illustrates dichotomous branching and the air exchange zones. Step 2 shows the impedance profile of the dichotomous branching based on the volume of exchanged air. Step 3 associates the impedance profile to the branching pattern using the maximum (red $\boldsymbol{\Delta}$ ) and minimum (green $\boldsymbol{\Delta}$ ) and the gradients of the inverted impedance profile.

EIT image, the enhanced resolution is equivalent to 8 additional electrodes i.e., 24 electrodes on the belt.

\section{B. EIT Plane Decorrelation}

In this section, the method for projected $3 \mathrm{D}$ to $2 \mathrm{D}$ AT extraction based on EIT cross-sectional plane decorrelation is outlined. Fig. 5 shows the three EIT plane decorrelation steps to accurately identify the branching trajectories from the EIT impedance matrix distribution. In step 1 the correlation between the volume of air exchange that is a function of the branching pattern and its geometrical features is computed. In step 2 the relations of the superimposed impedance distribution and branching profile are clarified. In step 3 the branching profile is used to trace the branching centerlines. The tracing engine utilizes the branching information in an enhanced EIT image $\mathrm{f}^{\prime}(x, y)$, namely connecting nodes $(\mathrm{CNs})$ and airflow field $\left(A F_{\theta}\right)$ for AT detection. Steps 1 and 2 below describe the principles relating to the impedance profile and AT. Selection of CNs and computation of $A F_{\theta}$ are detailed in step 3.

\section{Step 1. Air Exchange and Tree Pattern}

This section explains the amount of air exchanged using the dichotomous branching model [17]-[19] as shown in step 1 of Fig. 5. The volume of air exchanged at the termination nodes (i.e. alveoli zones) of the branching is a function of the geometrical features of the tree pattern including the branching length $(L)$, angle $(\theta)$ and diameter $(D)$. Therefore, the overall exchanged air volume which is seen as the captured impedance in the EIT cross-sectional plane is proportional to the area $(A=$ $L \cdot D)$ and air velocity $(\mathrm{Vel})$ in each segment of the tree. The symmetrical dichotomous branching model example in step 1 consists of one branching layer (i.e. $D_{0} \rightarrow D_{1}$ or $D_{0} \rightarrow D_{2}$ ). The stem branch $\left(D_{0}\right)$, termed "parent" is divided into two "daughter" sub-branches $\left(D_{1}\right.$ and $\left.D_{2}\right)$. This process can be repeated a number of times to form a hierarchical dichotomous division. For instance, the diameter of the parent $D_{0}$ can be

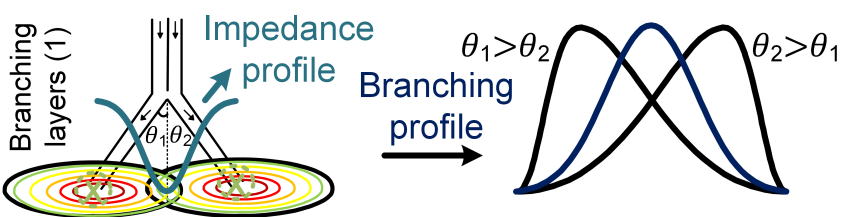

(a)
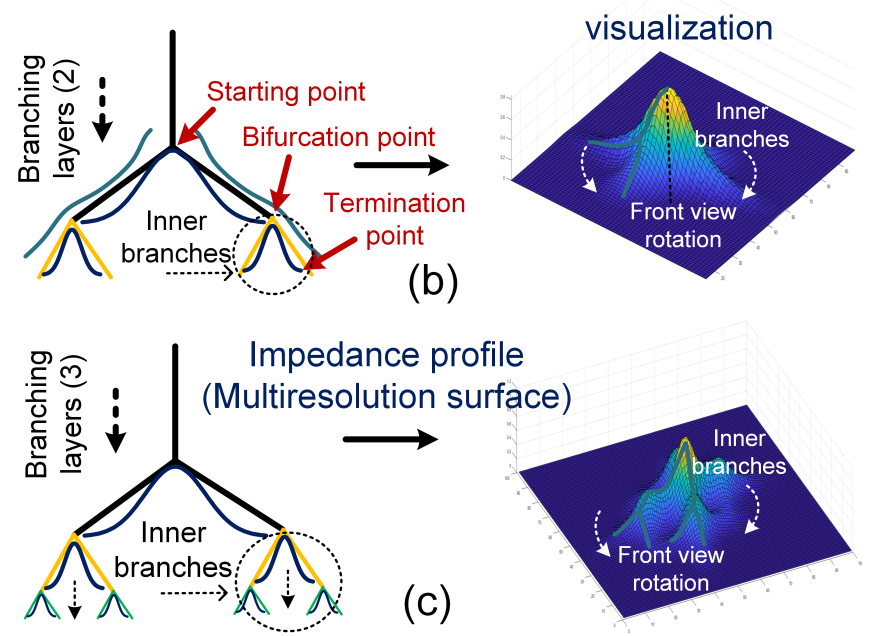

Fig. 6. Illustration of impedance superposition and branching profile. (a) Branching profile of a simple dichotomous branching. The superposition of the impedance at injection nodes (inj1 and inj2) define the effective branching profile. (b) Impedance profile visualization of two branching layers. The outer branches are aligned with the right half of the visualized surface and the inner branches are rotated to the left half of the surface. (c) Three-layer branching. Added branching layers result in multi-resolution surface quantization emulating EIT impedance captured on its cross-sectional plane.

related to the diameters of the daughters $D_{1}$ and $D_{2}$ by [17]:

$$
D_{0}^{x}=D_{1}^{x}+D_{2}^{x}
$$

where $x$ defines the flow regime and typically lies somewhere between 2.333 and 3.0 for intermediate flow. The branching length ratio varies around a constant value $L_{k}$, such that $L_{0} / L_{1}$ or $L_{0} / L_{2}=L_{k}$ [20]. The volume of airflow of a long branch is the sum of smaller branches. The impedance distribution on the EIT plane is effectively correlated with the volume of air exchanged indicated by the dotted green circles in step 1 of Fig. 5 which are a function of the branching geometrical characteristics.

\section{Step 2. Impedance Superposition and Branching Profile}

As noted earlier, the EIT cross-section plane captures the regional activity in each lung which is the effect of superimposed injected air volume at different nodes and directions. The key principle in identifying the correlation between the branching signature and impedance profile is superposition. Three branching profiles are considered in Fig. 6 for visualization purposes. The first profile in Fig. 6(a) is the simplest dichotomous branching. The impedance is high at the injection nodes (inj1 and inj2) as the EIT projects the volumetric ionized air volume to the plane (the impedance zones are shown with contour profiles where the dark red and green correspond to the highest and lowest amplitude variations, respectively). The impedance profile shows the 

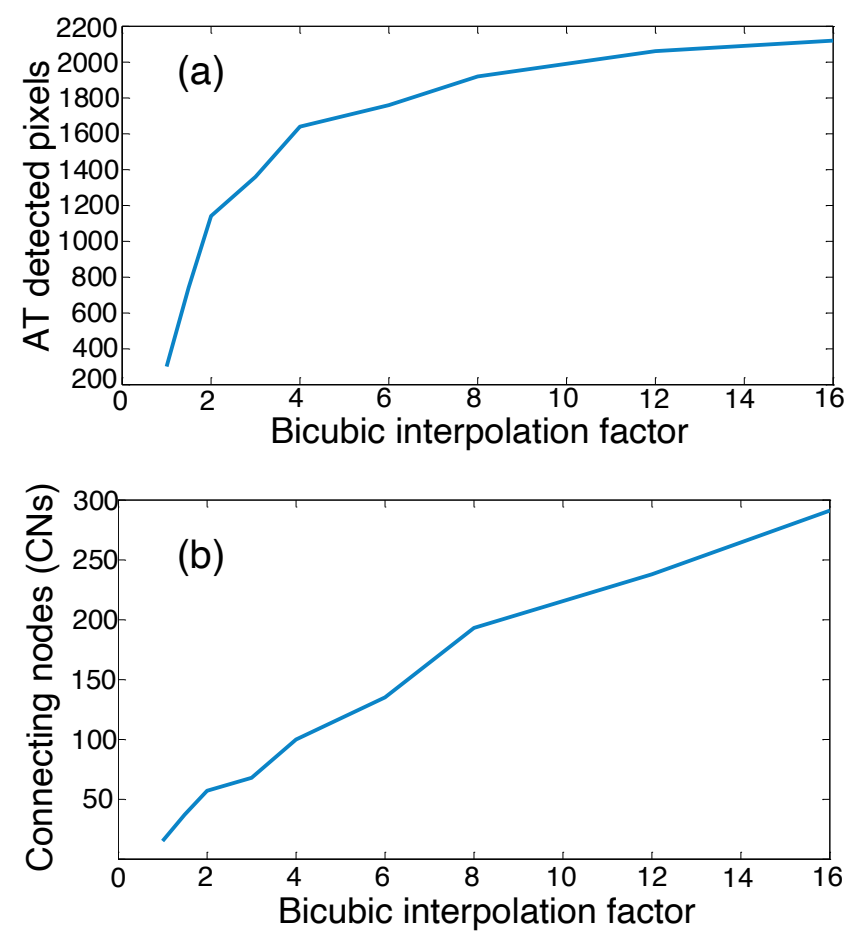

Fig. 7. (a) Tradeoff between detected centerline pixels versus image grid size $\left(N_{\text {Size }}\right)$. (b) Identified source and sink nodes (extrema) versus image grid size. The grid size is manipulated using bicubic interpolation factors 1 to 16 (bicubic interpolation factors 2, 8 and 16 correspond to grid sizes $64 \times 64$, $256 \times 256$, and $512 \times 512$ respectively).

amplitude variations across the injection zones in Fig. 6(a). The maximum points are aligned with the injection nodes and as it goes to the right from inj1 (or to the left from inj2) the impedance amplitude is attenuated. The midpoint of the profile shows the minimum which is associated with the lowest superimposed impedance level. The branching signature (centerline coordinates, start and end points) can be traced by the inverted impedance profile [i.e. the branching profile in Fig. $6(\mathrm{a})]$. By identifying the gradient of the branching profile and the connecting nodes, the branching signature can be traced. Certain characteristics can be identified from the detected $\mathrm{CNs}$ shown in the left side of Fig. 6(b): 1) The source nodes (maxima in red) are the start of branching [i.e. starting points (SPs)]. 2) The sink nodes (minima in green) are the termination points of the branches (TPs). 3) Dichotomous branching from SP or TP is interpreted as a bifurcation point (BP). The impedance profile representing the changes in the branching angles $\left(\theta_{1}\right.$ and $\left.\theta_{2}\right)$ is shown in Fig. 6(a) for the simple and symmetrical branching $\left(\theta_{1}=\theta_{2}\right)$, and the dichotomous or asymmetrical branching $\left(\theta_{1} \neq \theta_{2}\right)$. Changes in branching geometrical features (angle, diameter and length) result in branching profile skewness.

The second profile in Fig. 6(b) has two branching layers. Their impedance profile cannot be simply illustrated as a front view model; thus, surface visualization is shown for impedance superposition purposes. It can be seen from the visualized surface that the inner branches are rotated to the right side of the surface after impedance superposition. The generated surface branching profile is based on a rotated and scaled version of the dichotomous model (or AT anatomical model [17]). The superposition can be repeated based on the anatomical branching principles yielding a multi-resolution
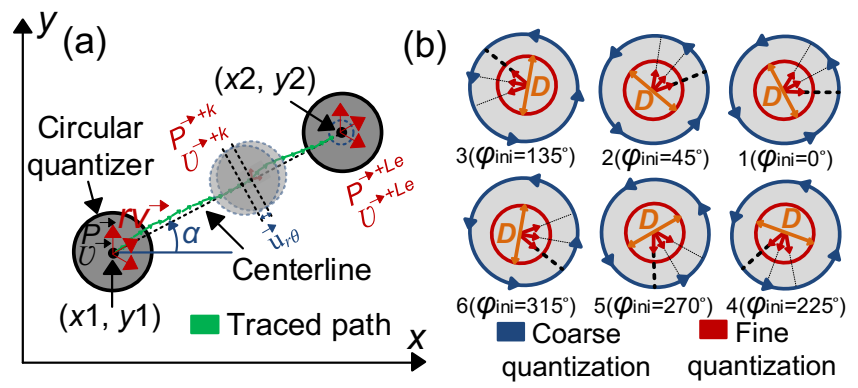

Fig. 8. (a) Tracing a possible path between two endpoints, starting at $\vec{p}_{(x 1, y 1)}$ with initial direction of $v^{\rightarrow}$ to $p_{(x 2, y 2)}^{\rightarrow+l e}$ where $l e$ is the length of segment. The designed circular quantizer discretizes the airflow field orientations with respect to the computed centerline (dotted black line) in segment tracing. (b) The coarse and fine quantization is illustrated at different angles relating to different discretization initiations.

quantized surface as shown in Fig. 6(c). The impedance level is a function of the number of branching layers, populations, asymmetry (i.e. $\theta_{1}>\theta_{2}$ or $\theta_{1}<\theta_{2}$ ), geometrical features and directions.

Step 3. Selection of CNs and Gradient Computation

Although an EIT image is upscaled using bicubic interpolation from $32 \times 32$ pixels to an arbitrary size, image quality is still not sufficiently detailed to accurately detect airflow and the AT branches. Embedding multiscale information to the upscaled EIT image using RQM [15] increases the probability of branch detection as shown in Fig. 7 but at a higher computational cost which is proportional to the number of arithmetic operations [21]. An enhanced upscaled image $\mathrm{f}^{\prime}(x, y)_{128}$ is considered sufficient for branch detection in real-time FI calculation (see Section III.C.3). As shown in step 3 of Fig. 5, the tracing of a branch is continued, quantifying the local maxima and minima in the image and the gradient of the branching profile. The branching gradient is expressed as [22]:

$$
A F_{\theta}=\tan ^{-1}\left(\frac{\partial \mathrm{f}^{\prime}(x, y)_{128}}{\partial y} / \frac{\partial \mathrm{f}^{\prime}(x, y)_{128}}{\partial x}\right) \text {. }
$$

where $\partial \mathrm{f}^{\prime}(x, y)_{128} / \partial y$ and $\partial \mathrm{f}^{\prime}(x, y)_{128} / \partial x$ are the derivatives of $\mathrm{f}^{\prime}(x, y)_{128}$ with respect to the $x$ and $y$ directions.

\section{AT Tracing Algorithm}

This section first outlines the tracing algorithm utilizing a circular quantizer and AT branching principles. The AT branching validation using high resolution computed tomography (CT) ground-truth is described in Section III.C.3.

\section{1) Directional Self-exploratory Tracing Operator}

An automated self-exploratory algorithm is proposed to detect the origins of an AT network embedded in the enhanced EIT image $\mathrm{f}^{\prime}(x, y)$. The inputs to the operator are $M_{\text {dir }}$ (airflow direction matrix) and $M_{\text {ext }}$ (extrema matrix) which both contribute to the tracing initiation and direction of each branch, and the output of the operator is a network of the linked branches. An example of segment tracing is shown in Fig. 8(a). It is defined by its two endpoints $(x 1, y 1)$ and $(x 2, y 2)$ (source $\rightarrow$ sink). It starts from $(x 1, y 1)$, identifies the linking segments on $M_{d i r}$ and terminates at $(x 2, y 2)$. The tracing operator uses both coarse and fine angular quantization providing a directional 2D discretization. This allows to investigate the branching relations between the endpoints $(x 1, y 1)$ and 
$(x 2, y 2)$. In the coarse steps the relevant quantization quadrant provides a limit for defining the finer quantization steps. It is discretized to three values separated by $45^{\circ}$ as shown in Fig. $8(b)$. The rotation flexibility of the proposed tracing engine allows detection of branch fluctuation inconsistencies in $M_{d i r}$. The direction of the rotation vector $r \vec{v}$, red arrow in Fig. 8(a), is set by the angular discretization and the branching diameter is set by a left-right orange arrow $(\leftrightarrow)$ in Fig. 8(b) which is investigated further in this section. The flexibility of the proposed operator can be achieved by using a rotation vector over a coarse discretization limit $\left(\varphi_{c o}\right)$ :

$$
r v_{r \theta=0,1,2}=\varphi_{\text {ini }+}\left(r \theta \times \Delta \varphi_{\mid \Delta \varphi=45^{\circ}}\right) .
$$

Equation (7) shows the direction of $r v^{\rightarrow}$ initiated at $\varphi_{i n i}$ and quantized within the coarse quadrant $\varphi_{c o}$ (denoted by $r \theta$ index $0,1,2)$. To assist the tracing engine with reliable branch detection, a centerline is defined between points $(x 1, y 1)$ and $(x 2, y 2)$. The quantization process is associated with the black dashed line centerline path shown in Fig. 8(a). This is a valid assumption since in the AT anatomical model a branching segment typically shows little deviation when linking source to sink nodes. Starting from the initial coordinates $(x 1, y 1)$, point $p \rightarrow$ and initial direction $v^{\rightarrow}$, the branch direction is traced by iterative search in $M_{\text {dir }}$ of the next point location $p^{\rightarrow+1}$ and direction $v^{\rightarrow+1}$ in a feed-forward manner. The start tracing point $p^{\rightarrow}$ is set to the highest peak in the left (or right) lung and the initial direction $v \rightarrow$ corresponds to the centerline angle $\alpha$ between the chosen endpoints $(x 1, y 1)$ and $(x 2, y 2)$, where:

$$
\alpha=\arctan \frac{\left(\Delta y=y_{2}-y_{1}\right)}{\left(\Delta x=x_{2}-x_{1}\right)} .
$$

The location of the new point $p^{\rightarrow+1}$ is estimated as:

$$
p^{\rightarrow k+1}=p^{\rightarrow k}+\overrightarrow{\mathrm{u}}_{r \theta}
$$

where the $\overrightarrow{\mathrm{u}}_{r \theta}$ is the jump-ahead oriented unity vector. In the coarse phase, only a specific circular region is chosen and discretized into three values. $\overrightarrow{\mathrm{u}}_{r \theta}$ is computed based on the angular quantizer response which has the least amount of deviation from the centerline and the maximum consistency to the direction history $\left(v^{\rightarrow}\right)$ as:

$$
\overrightarrow{\mathrm{u}}_{r \theta}=\underset{r v_{r \theta \in\{0,1,2\}}}{\operatorname{argmin}}\{\underbrace{\left\{M_{\text {dir }}\left(r v^{\rightarrow}\right)-\alpha\right\}}_{\text {Deviation }}-\underbrace{v^{\rightarrow}}_{\text {History }}\} .
$$

As shown in Fig. 8(b), the orange left-right arrow defines the branching diameter $(D)$. First, the initial diameter $\left(D_{0}\right)$ is computed and then the branching diameter for each branching layer (daughter) is set to $\left(D_{1}\right.$ or $D_{2}=D_{0} / \sqrt[x]{2}$ if $\left.D_{1}=D_{2}\right)$ [17] where $x$ defines the flow regime; in the tracing algorithm $x=$ 2. The branching diameter can be generalized as $D_{b l=1 \ldots 6}=D_{0} /(\sqrt[x]{2})^{b l}$, where $b l$ is the number of branching layers (which has a maximum value of 6 in this design). The branching template on each side, right $T_{R}(x, y)$ and left $T_{L}(x, y)$, perpendicular to the extracted tree can be expressed as:

$$
\left\{\begin{array}{l}
\left.T_{R}(x, y)\right|_{k=1 \ldots l e}=\sum_{R o} \sum_{C o} p^{\rightarrow k}(x+R o, y+C o) \\
\left.T_{L}(x, y)\right|_{k=1 \ldots l e}=\sum_{R o} \sum_{C o} p^{\rightarrow k}(x+R o, y+C o)
\end{array}\right.
$$

where $l e$ is the length of the traced tree segment $R o$ and $C o$ define the size of the template extractor matrix perpendicular to the traced path. The extracted left-right templates are exploited to define the optimal branching diameter $\left(D_{\text {opt }}\right)$ as:

$$
\rho_{1}\left|T_{R}(x, y)-T_{L}(x, y)\right|>D_{\text {opt }}>\rho_{2}\left|T_{R}(x, y)-T_{L}(x, y)\right|
$$

where $\rho_{1}$ and $\rho_{2}$ are threshold coefficients whose values are set empirically based on the grid size $\left[\mathrm{f}^{\prime}(x, y)\right]$. Equation (12) presents the fact that sweeping of $\rho_{1}$ and $\rho_{2}$ can lead to sparse $\left(D_{S p}<D_{\text {opt }}\right)$ and dense $\left(D_{\text {Den }}>D_{\text {opt }}\right)$ branch tracings where $D_{S p}$ and $D_{D e n}$ are the sparse and dense branching diameters. In sparse branch extraction, the signature of the branch structure is not well reflected and in dense branch extraction the probability of branch overlap is high.

The tracing process between the endpoints $(x 1, y 1)$ and $(x 2, y 2)$ is terminated if the following three conditions are satisfied: 1) If any $\mathrm{CNs}$, local peak or valley, are outside the considered effective lung area. 2) The tracing process is terminated when $p^{\rightarrow+1}$ is seen within a termination threshold $(\zeta)$ of $(x 2, y 2)$ or $p^{\rightarrow+1}=\left(x_{2} \pm \zeta, y_{2} \pm \zeta\right)$; $\zeta$ is set between 1 and 3 by experiment. In the case of false tracing, the extraction phase is not completed, $\zeta$ is tuned adaptively between 1 and 3 for completion of the traced path. 3) The initial assumption is that a local peak can have multiple paths toward the various local valleys. However, if there are some paths which start from the local peak [e.g. $(x 1, y 1)]$ and encounter unacceptable deviations that result in false path tracing, they are identified as "false branching". The deviation in the algorithm occurs when it exceeds the following limit:

$$
\underset{r v^{\rightarrow} r \in \in\{0,1,2\}}{\operatorname{argmin}}\left\{M_{d i r}\left(r v^{\rightarrow}\right)-\alpha\right\}>60^{\circ} .
$$

\section{2) Convergence of Branches}

a) AT in Lungs:

The process of finding the partial trees in the lungs is the layered monitoring scheme (LMS). In LMS, each layer corresponds to the local peak of the left or right lungs. The local peak with the highest value is selected and the local valleys around it are monitored. The process of monitoring the local valleys uses the $J_{G}(x, y)$ matrix defined as:

$$
J_{G}(x, y)=\sum_{x \text { Peak }-p}^{x \text { Peak } p} \sum_{y \text { Peak }-p}^{y \text { Peak } p} M_{\text {ext }}\left(x_{\text {peak }}, y_{\text {peak }}\right)
$$

where $p$ defines the size of the monitoring window for the local valleys and is defined based on the peak locations $\left(x_{\text {peak }}, y_{\text {peak }}\right)$ with respect to the effective lung border $\left(r_{e f f}\right)$. To obtain $r_{e f f}$, a common approach is to estimate the boundary from an anatomic model based on an estimation of the patient chest size or a CT if available. It is also possible to extract the boundary information from a probabilistic approach [23] which automatically selects the best estimated forward model fit from pre-stored library models.

As shown in Fig. 9, the radius of the initiated kernel is increased by a fixed (or variable) step size and the local valleys are saved in the associated layer [e.g. layer 1] for branch tracing sequencing. In Fig. 9, an example is shown of the formation of a partial tree by branch tracing between the first local peak $\left(x_{\text {peak } 1,}, y_{\text {peak } 1}\right)$ and the detected valleys in layer $2\left(x_{L 1,2}, y_{L 1,2}\right)$, layer $4\left(x_{L 1,4}, y_{L 1,4}\right)$ and layer $5\left(x_{L 1,5}, y_{L 1,5}\right)$. Defining $p$ based 


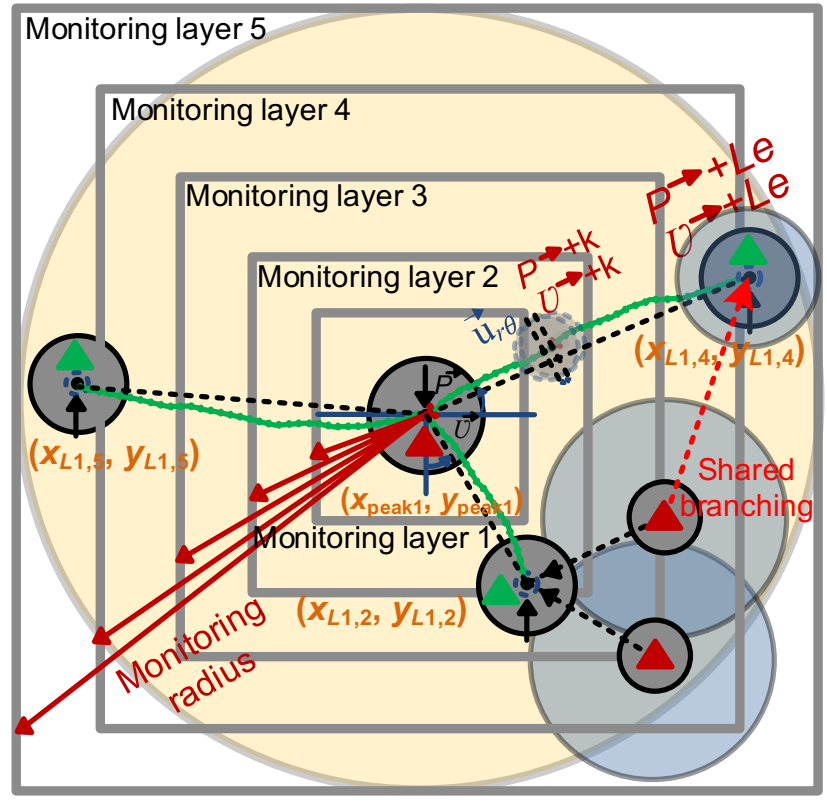

Fig. 9. The proposed layered monitoring scheme for extraction and linking of the partial trees. As illustrated in the figure, the monitoring radius is adaptively increased to find the sink nodes (green $\boldsymbol{\Delta}$ ). The maximum monitoring radius (orange and blue monitoring regions) are defined by the lungs' effective borders $\left(r_{e f f}\right)$. In this example, there are three paths traced by circular quantizer on airflow field $\left(A F_{\theta}\right)$.

on the location of the source node with respect to $r_{e f f}$ allows assigning of a higher probability weight to the identified valleys in the monitoring region. This helps to avoid shared branching between the partial trees as shown in Fig. 9.

In the tracing process, the coordinates of each branch are saved, and a location matrix $\left(L_{\text {Matrix }}\right)$ is automatically generated. The process is repeated for all detected local valleys until all probable segments with respect to the first local peak have been highlighted and numbered in $L_{\text {Matrix }}$. After extraction of all the branches associated with the local peak $\left(x_{\text {peak } 1}, y_{\text {peak } 1}\right)$ in the monitoring layers (e.g., layer $1 \ldots$ layer 5 ), the next possible peak is addressed sequentially and follows the same procedure. The ability to "jump" between the local peaks introduces the partial tree extraction concept. $L_{\text {Matrix }}$ consists of connected sub-trees forming different layers and provides information regarding branching; starting points (SPs), bifurcation points (BPs) and termination points (TPs).

\section{b) Labeling and Intersection Monitoring:}

Together with $L_{\text {Matrix }}$, a labeling matrix $\left(L_{C N S}\right)$ is generated to link the partial trees and store the information about the relationship between the branches and the CNs. An ordering scheme is adopted for labeling the traced branches for each local peak. The number of each layer is considered as the index of branching $\left(k_{L n, B i}\right)$ where $L n$ and $B i$ are the layer number and branch index. The generated network represents the AT structure. As the tracing proceeds, each branch labeling information is used to evaluate the possibility of intersection with other branches.

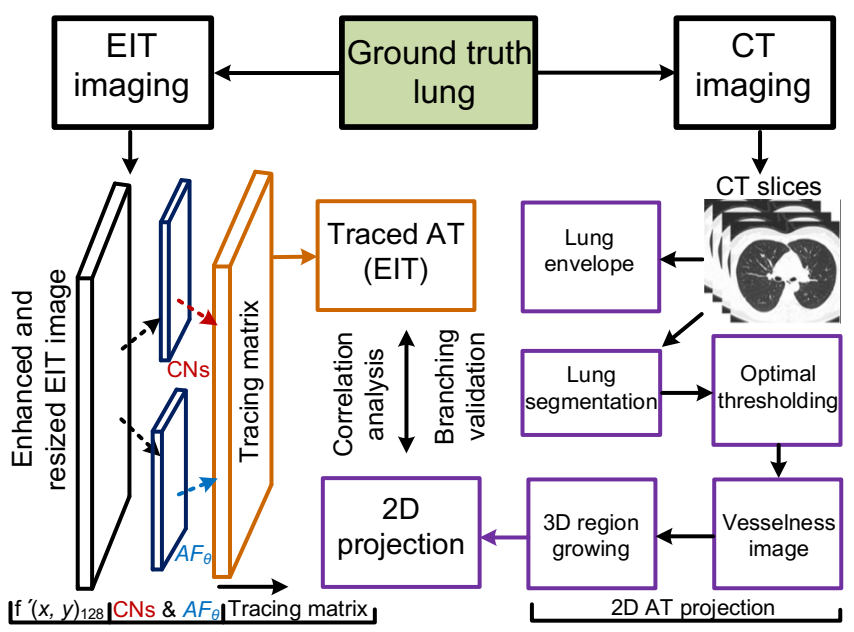

Fig. 10. Proposed validation set-up. Both CT and EIT paths utilizing the same lung as ground truth. The CT path employs 3D segmentation methods in [24][26]. The EIT path first performs derivation of features $\left(\mathrm{CNs}\right.$ and $\left.A F_{\theta}\right)$ for tracing AT in EIT image and subsequently traces the AT. The traced AT and $2 \mathrm{D}$ projection of AT from $\mathrm{CT}$ path are used for template matching and validation analysis.

\section{3) Branching validation from images of a patient lung:}

To assess the efficacy of the proposed tracing algorithm the setup shown in Fig. 10 is proposed. Tracing the AT in the lungs uses static EIT images and CT images from an established ground truth ${ }^{1}$. Both EIT and CT paths use the same lungs for imaging. The CT path utilizes the slice-by-slice CT segmentation and automated detection of the complete 3D AT [24]-[26]. CT slices covering the whole lung were used as reference.

Fig. 11(a) shows the 3D reconstructed model of AT in a patient with collapsed region in the left lung. For validation purposes, the branching profile of the projected 3D lung model to the cross-sectional (or transverse) plane and its corresponding centerline coordinates are shown on the left side of Fig. 11(d) from the viewpoint indicated on top of Fig. 11(a). The Sentec EIT Pioneer Set (Sentec AG, Switzerland) [27] was used to measure the impedance distribution with 32 electrodes. The surface potential differences were reconstructed using GREIT software [28] with $32 \times 32$ pixel resolution. The captured EIT image was fed to the resolution enhancement and the $\mathrm{f}^{\prime}(x, y)$ was sent to the pre-tracing units for computing $M_{\text {ext }}$ (CNs) and $M_{\text {dir }}\left(A F_{\theta}\right)$ as shown in Fig. 11(b). The $A F_{\theta}$ and CNs are illustrated using the quiver plot with directional black arrows and red-green markers $\boldsymbol{\Lambda} . M_{\text {ext }}(\mathrm{CNs})$ and $M_{\text {dir }}\left(A F_{\theta}\right)$ were then fed to the iterative tracing algorithm, Fig. 11(c), to perform partial and global AT extraction. The AT traced by the algorithm are color-coded lines with the embedded $D_{\text {Opt }}$ that is reduced according to the colored branching layers (layer 1...layer 5).

Two points need to be taken into consideration during the branching iterations: 1) Since the algorithm utilizes reverse processing when tracing the true branches, there are numerous initial branching possibilities and most of them are removed in steps 1 and 2 in Fig. 11(c). 2) The branching is only valid within

https:/clinicaltrials.gov/ct2/show/NCT02962505?term=NCT02962505\&rank $=1$
${ }^{1}$ Ethics approval link for the clinically captured EIT and CT images:
ClinicalTrials.gov; Identifier: NCT02962505. 


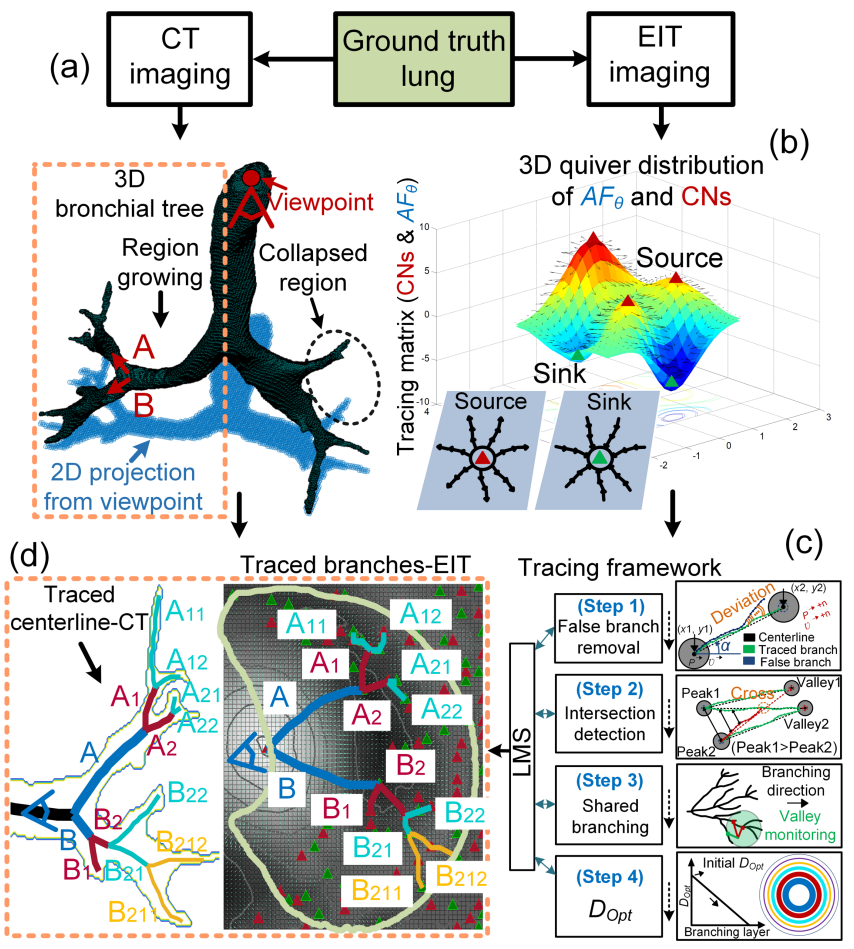

Fig. 11. (a) 3D reconstructed ground truth using CT images from the viewpoint. 2D projection of the lungs from the viewpoint is also shown using scatter plot in this figure. The 3D model shows collapsed regions on the left lung. (b) Illustration of 3D airflow distribution using quiver plot. The seed points, source (red)) and sink (green) are annotated on the figure. (c) The iterative validation steps in the proposed AT tracing framework that employs the CNs and $A F_{\theta}$ matrix information. (d) The 2D projection model of the traced branches on cross-sectional (transverse) plane using CT and EIT data. The branching pattern in CT-EIT show accurate alignment from the nodes A and $\mathrm{B}$. Labelling of the branches (e.g. $\mathrm{B}, \mathrm{B}_{2}, \mathrm{~B}_{22}$ and $\mathrm{B}_{212}$ ) shows the alignment of the branching between CT-EIT paths.

the effective lung border. The traced branching profile from the EIT path shows an acceptable directional and spatial correlation with the 2D AT from the CT path as interpreted by the displayed labels in Fig. 11(d) (e.g. A, $A_{1}, A_{11}$ and $A_{12}$ ). It should be noted that partial branching misalignment results in missing a small percentage of the branching tree pixels; this, however, has negligible effect on the FI calculations.

Fig. 12 shows the branching template resolution and normalized cost versus the grid size using sweeping bicubic interpolation. The branching template resolution (BTR) is defined as $\mathrm{BTR}=\mathrm{TDB} /(\mathrm{TDB}+\mathrm{FB}+\mathrm{MB}) \times 100$, where TDB is the number of truly detected branches, FB (false branches) are those due to randomly superimposed artefacts and $\mathrm{MB}$ is the number of both missed minor and major branching segments between the reference CT and the traced EIT ATs. Five of the total number of identified branch segments were labeled as major ATs since their diameters were large and 16 of them were labeled as minor AT segments, resulting in overall BTR of $77.3 \%$ at $\mathrm{f}^{\prime}(x, y)_{128}$ compared with the CT 2D branching reference. There were one major $\mathrm{FB}$ and five minor MBs. It can be seen from Fig. 12(a) that a much lower correlation percentage is obtained when only the resized EIT image without RQM is used for estimation of the branching area. On the other hand, a higher correlation is achieved when the EIT image is resized, and resolution enhancement is embedded into the image [Fig. 12(b)]. The simulations show
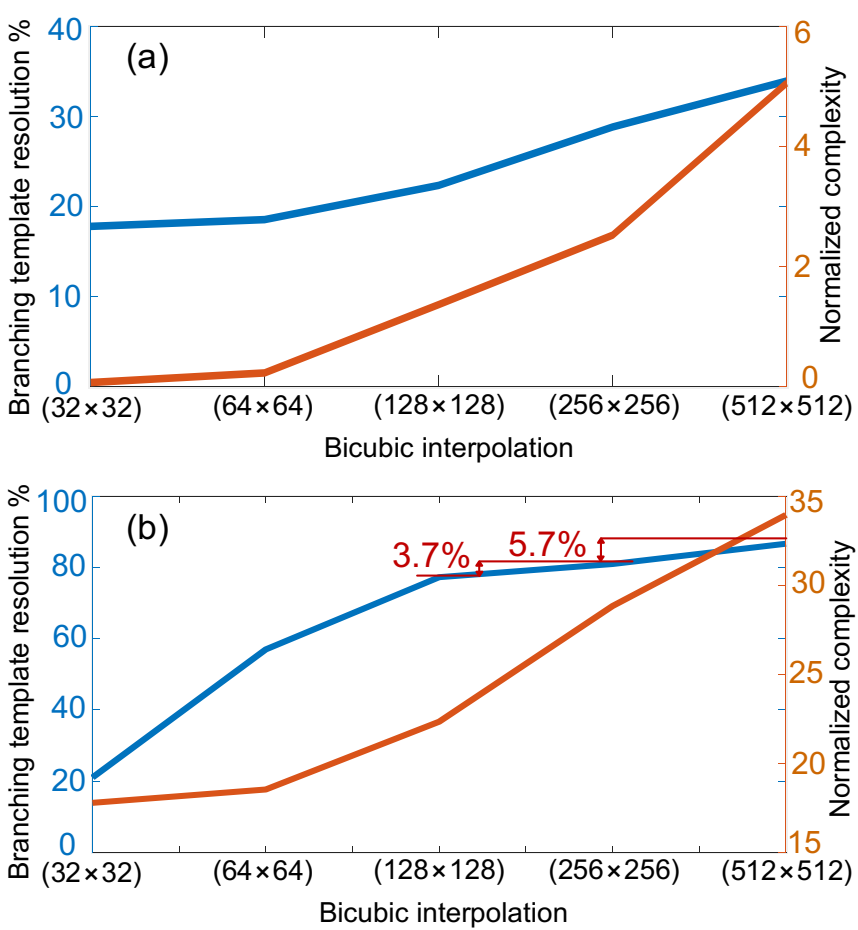

Fig. 12. Branching template resolution versus image grid size and the normalized complexity; (a) without RQM and (b) with RQM. In this analysis, the branching template resolution is the function of image grid size and resolution enhancement method. The grid size is manipulated using bicubic interpolation factors $1,2,4,8$ and 16 corresponding to grid sizes $32 \times 32$, $64 \times 64,128 \times 128,256 \times 256$ and $512 \times 512$, respectively. The red plot demonstrates the normalized complexities of different grid sizes used for airway tree extraction.

that the branching template resolution between $\mathrm{RQM}_{128 \times 128}$ $\left[\mathrm{f}^{\prime}(x, y)_{128}\right]$ and $\mathrm{RQM}_{512 \times 512}$ is less than $9.5 \%$ which is a good candidate for real-time FI calculation. $\mathrm{RQM}_{256 \times 256}$ and $\mathrm{RQM}_{512 \times 512}$ are more aligned when tracing multi-layer and finer dichotomous branching, but with almost twice the computational cost compared to $\mathrm{RQM}_{128 \times 128}$.

The complexity plots in Fig. 12(a) and (b) show that the computational cost of the system increases almost exponentially with respect to the size of the image. The qualitative evaluation of the proposed AT tracing algorithm was also examined with EIT images with noise added to the generated raw EIT voltages $\left(v_{r}\right)$ by superimposing white Gaussian noise $\left(\sigma_{N}=0.025,0.05\right.$ and 0.075$)$ [15]. The results indicate that the algorithm underestimates the BTR of a nonnoisy image by only $0.6 \%$ in the worst-case scenario. This low error value is associated with the use of local statistics in the RQM for multiresolution enhancement; thus, the image details are enhanced, and image noise is suppressed providing noise robustness in tracing the ATs.

\section{ACCURACY OF FI RESULTS}

\section{A. EIT Data Generation for FI Calculation}

In order to compare the performance of the proposed method for FI calculation with other methods, EIT images with known FIs are required from clinical data. Real EIT images with FI indices assessed by an expert are possible, but the accuracy of human interpretation may not be sufficient compared with 


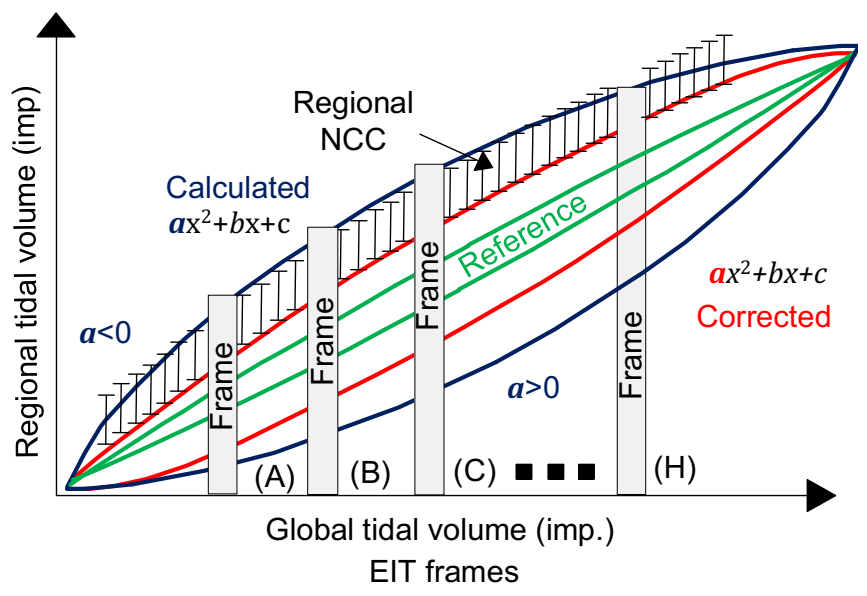

Fig. 13. Global impedance waveform of a single breath. Positions (A-H) correspond with the global integral impedance $\left(Z_{\mathrm{GL}}\right)$ computed by the appropriate resistive network representing specific inhomogeneity level. The reference (ground truth) FI coefficients in quadrants for the generated single breath were computed using polynomial $y=a x^{2}+b x+c$.

artificial image generation. An alternative is the regional lung injury injection [5] which provokes ventilation inhomogeneity to estimate regional filling capacity of the lung quadrants. However, EIT image generation using this approach is timeconsuming and unsafe, and in practice results in the generation of a few images only, limiting the degree to which the accuracy of an algorithm could be assessed.

The conventional approach [29] to create EIT images uses a flat cylindrical tank with electrodes attached around it, filled with conductive water. Conductive (metal) and resistive (plastic) objects are placed in the tank to emulate the lungs functionality. This method offers low spatial resolution tuning capability in emulating lung functionality. Tuning capability sets the non-uniformity factor in ventilation. To enhance the tuning capability, multiple phantoms with different sizes and in different configurations are required over the ventilation phase which is a complex task.

The Sentec EIT Pioneer Set provides a test phase for artificial image generation based on resistive phantoms [30]. In the phantom test phase, an algorithm adjusts the resistive inhomogeneities to emulate the overall breath rate, breath amplitude, and independent breathing of the left or right lungs. The real-time EIT images are displayed on a PC screen enabling adjustment and fine-tuning of the desired inhomogeneities. Adjustment of these parameters can provide sufficient diversity of data for detailed assessment of different AT algorithms.

Since the traced AT model and the captured EIT image are correlated, the AT structure including geometrical features and asymmetry properties can vary based on the defined regional resistive distribution in the mesh phantom. The set-up generates two outputs:

1) Relative FI coefficients for each lung quadrant in the ventilation phase. These coefficients are used for accuracy assessment and are computed from the appropriate resistive network configurations. In Fig. 13, the ventilation phase is modeled in eight frames (A...H) each corresponding to a set-up for the desired inhomogeneity. It shows the FI coefficients corresponding to the eight frames based on traced AT conventionally calculated $a$ in blue, the corrected $a$ in red, and the reference $a$ in green.

2) A set of reconstructed EIT images in a $32 \times 32$ pixel matrix. These are fed to the FI calculation unit. The Sentec EIT Pioneer Set creates EIT images with different degrees of inhomogeneity for each quadrant. Four FI graphs emulating the regional-global impedance variation in each quadrant during respiration are derived using the generated $32 \times 32$ pixels EIT images. The calculated FI graphs and their associated polynomial filling index are shown in blue in Fig.14. The four datasets are $\mathrm{q}(\mathrm{RA} \rightarrow \mathrm{LP})$-Low1, $\mathrm{q}(\mathrm{RA} \rightarrow \mathrm{LP})$-Low2, $\mathrm{q}(\mathrm{RA} \rightarrow \mathrm{LP})$-High1 and $\mathrm{q}(\mathrm{RA} \rightarrow \mathrm{LP})$-High2, where 'Low' and 'High' denote the inhomogeneity intensity of lead or lag in the quadrants $(\mathrm{RA} \rightarrow \mathrm{LP})$.

\section{B. FI Results and Discussion}

Tracing of the AT provides the non-uniform correction coefficients (NCC) for the filling behavior of the lungs during spontaneous breathing. The degree of non-uniformity depends on the branching population in each lung quadrant. The NCC provide calibration of the $\mathrm{FI}$ in the ventilation phase so effective branching paths can be adaptively obtained for individual lung quadrants. The average NCC (ANCC) in each quadrant during ventilation is:

$$
\text { ANCC }=\frac{1}{\text { length }(\text { phase })} \sum_{V \text { phase }=A}^{\mathrm{H}} \frac{\mathrm{Z}\left(\text { qbArea }_{V \text { phase }}\right)}{\mathrm{Zq}_{V \text { phase }}}
$$

where $\mathrm{Zq}_{V \text { phase }}$ is the impedance integral of a quadrant and $\mathrm{Z}\left(\mathrm{qbArea}_{V \text { phase }}\right)$ is the corresponding impedance of the quadrant of the interpreted AT traced by the proposed tracing algorithm over the ventilation phase (Vphase). Both $\mathrm{Zq}_{V \text { phase }}$ and $\mathrm{Z}\left(\mathrm{qbArea}_{V \text { phase }}\right)$ consider the impedance integral within the defined lung border. ANCC is the average correction factor acquired over the ventilation cycle and it non-uniformly attenuates the regional deviations with respect to the generated reference $\left(\mathrm{FI}_{\text {Corrected }}=\mathrm{FI} \times \mathrm{ANCC}\right)$. In Fig. 14(a), the blue curve is calculated from $\mathrm{Zq}_{V \text { phase }} / \mathrm{Z}_{\mathrm{GL}}$ of the captured frames (A-H) over the ventilation phase. Applying the ANCC to the blue curve results in the red curve that has less deviation from the reference. The corrected graph has a red fitting coefficient $y=a x^{2}+b x+c$ that is close to the reference value.

To examine the regional collapse $(a>0)$ and hyperinflation $(a<0)$ during tidal breathing, the regional filling characteristics of the lungs of dataset 2 [q(RA $\rightarrow$ LP)-Low2] were calculated as shown in Fig. 14. It includes the calculated (blue), corrected (red) and reference (green) FI curves as well as the corresponding NCC graphs over frames A to $\mathrm{H}$. The fitting coefficients in all quadrants are computed from the resized original EIT image (blue curve) and the traced AT using the $\mathrm{f}^{\prime}(x, y)_{128}$ images (red curve). The polynomial fit in the red curve exhibits modified curvilinearity of lung respiration using the AT which reflects a more accurate interpretation of each region during spontaneous tidal breathing.

The regional filling characteristics of all the generated datasets $[\mathrm{q}(\mathrm{RA} \rightarrow \mathrm{LP})$-Low1, $\mathrm{q}(\mathrm{RA} \rightarrow \mathrm{LP})$-Low2, $\mathrm{q}(\mathrm{RA} \rightarrow \mathrm{LP})$ High1 and $\mathrm{q}(\mathrm{RA} \rightarrow \mathrm{LP})$-High2] using the polynomial fitting coefficients in the four lung quadrants (RA, LA, RP and LP) 
(a)

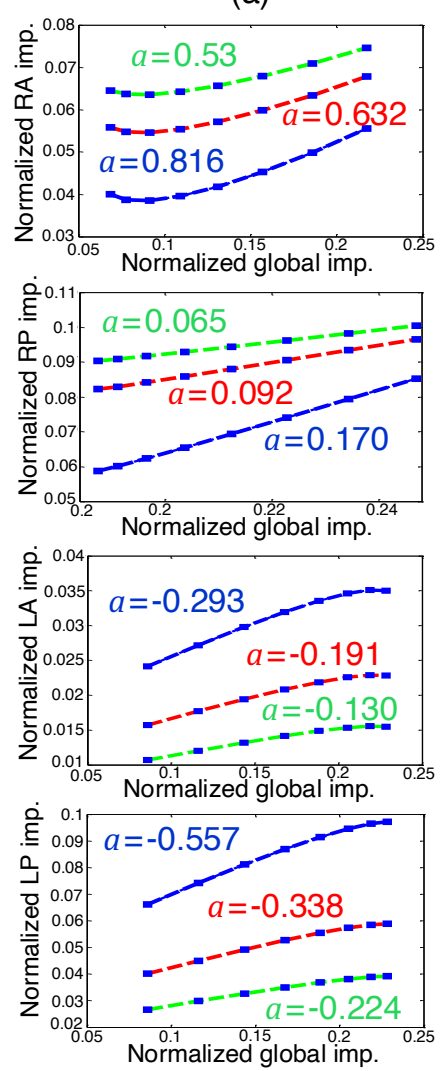

(b)
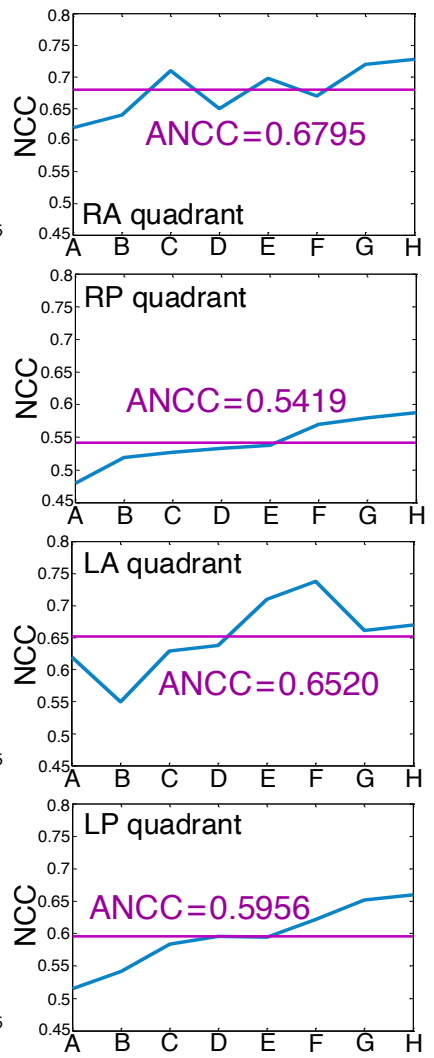

Fig. 14. (a) Second degree polynomial fit of regional right or left relative impedance change versus overall relative impedance change in $\mathrm{q}(\mathrm{RA} \rightarrow \mathrm{LP})$ Low2. (b) Plots show NCC in each respiration phase (A...H) and the overall $\mathrm{ANCC}=\left(\mathrm{NCC}_{\mathrm{A}}+\mathrm{NCC}_{\mathrm{B}}+\cdots+\mathrm{NCC}_{\mathrm{H}}\right) / 8$. ANCC emulates a reverse nonuniform skewed distribution for correcting the regional filling behavior.

were examined, and the results are summarized in Fig. 15 and Table I. In Fig. 15 the average correction of the polynomial coefficient for all the quadrants is $65 \%$. This percentage refers to the overall correction obtained by the traced AT over all the generated data. The three mean values are shown in green (reference), red (based on $32 \times 32$ standard EIT images) and the dark blue (based on the AT). According to the absolute deviation: $\mathrm{Y} 1=\operatorname{abs}\left(\right.$ mean $_{\text {green }}-$ mean $\left._{\text {blue }}\right)=0.2041$ and $\mathrm{Y} 2=\operatorname{abs}\left(\right.$ mean $_{\text {green }}-$ mean $\left._{\text {red }}\right)=0.0709$. The correction percentage is $\operatorname{abs}(\mathrm{Y} 1-\mathrm{Y} 2) / \mathrm{Y} 1) \times 100=65 \%$. $\mathrm{Y} 1$ is used in the denominator to enable examination of the corrected FI. Note that accurate assessment of the lung mechanics can be used for fine adjustment of recruitment manoeuvres to improve air exchange distribution in the lungs.

\section{C.Filling Index Analysis in a Clinical Setting Based on Standard EIT Images and Airway Tracing}

The regional filling capacity of the lungs of 10 subjects were measured from images provided by the Sentec 32 electrode EIT system during the CRADL ${ }^{2}$ project. The subjects were 10-28 months old and weighing 7-13 kg. The subjects were treated with either invasive ventilation or a high flow nasal cannula

${ }^{2}$ Ethics approval link for the CRADL project: ClinicalTrials.gov; Identifier: NCT02962505.

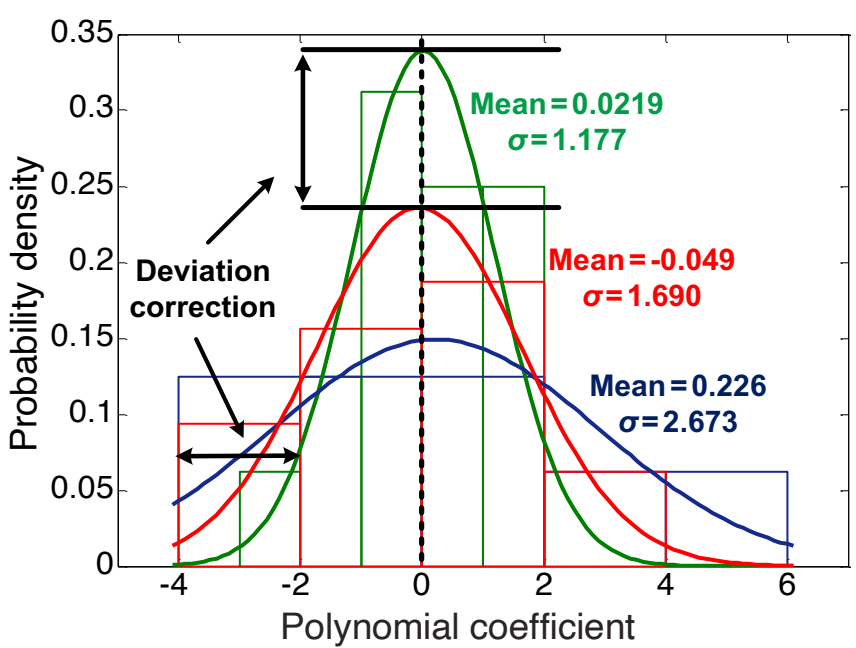

Fig. 15. Histogram results of regional FIs in all the generated artificial EIT images. FIs were calculated from plots of regional tidal volume versus global tidal volume obtained by EIT in lung quadrants. Histogram plots were fitted to normal distributions. Green: reference; Red: $32 \times 32$ based on standard EIT images (has lower deviation than reference); Blue: based on AT providing more accuracy.

Table I

Regional Polynomial Coefficients of Quadrants RA, LA, RP and LP in Generated Datasets Using the Sentec EIT Pioneer Set.

\begin{tabular}{|c|c|c|c|c|c|c|}
\hline \multirow{2}{*}{\multicolumn{3}{|c|}{ Datasets }} & \multicolumn{4}{|c|}{ Quadrants } \\
\hline & & & RA & LA & RP & LP \\
\hline \multirow{12}{*}{ Set-ups } & \multirow{3}{*}{ Low1 } & $\mathrm{A}^{\mathrm{a}}$ & -0.143 & -0.009 & 0.007 & 0.092 \\
\hline & & $\mathrm{B}^{\mathrm{b}}$ & -0.220 & -0.024 & 0.016 & 0.229 \\
\hline & & $\mathrm{C}^{\mathrm{c}}$ & -0.161 & -0.018 & 0.010 & 0.121 \\
\hline & \multirow{3}{*}{ Low2 } & $\mathrm{A}$ & 0.53 & -0.130 & 0.065 & -0.224 \\
\hline & & B & 0.816 & -0.293 & 0.170 & -0.557 \\
\hline & & $\mathrm{C}$ & 0.632 & -0.191 & 0.095 & -0.338 \\
\hline & \multirow{3}{*}{ High1 } & $\mathrm{A}$ & 1.119 & 1.310 & -0.926 & -1.582 \\
\hline & & B & 2.603 & 3.420 & -2.091 & -3.932 \\
\hline & & $\mathrm{C}$ & 1.509 & 1.853 & -1.261 & -2.162 \\
\hline & \multirow{3}{*}{ High2 } & $\mathrm{A}$ & -2.014 & 1.905 & -1.605 & 1.956 \\
\hline & & $\mathrm{B}$ & -2.677 & 4.975 & -3.62 & 4.853 \\
\hline & & $\mathrm{C}$ & -2.078 & 2.595 & -2.116 & 2.338 \\
\hline
\end{tabular}

${ }^{\mathrm{a}}$ Reference FI; ${ }^{\mathrm{b}}$ Calculated FI; ${ }^{\mathrm{c} C o r r e c t e d ~ F I . ~}$

(HFNC) to improve their lung functionality. Inhalation treatment used either Salbutamol or Ipratropium. The flow rates during inhalation and the time applied (3-5 minutes) were determined by the clinician based on each subject's condition. The EIT images were used to demonstrate the operation of the FI measurements. The FI measurements had three steps: 1) FI measured just before application of drugs on inhalation (pre); 2) Application of drugs on inhalation for periods determined by the clinician; 3) FI measured after the inhalation period (post). Measurements were performed on all the lung quadrants (RA, RP, LA and LP) of the 10 subjects. The FI derivation was calculated using the polynomial $y=a x^{2}+$ $b x+c$ between the end-expiratory point (Frame 1) to the maximal inhalation point (Frame 25) which is compatible with the transition over the inhalation period. For comparison the FI coefficients were measured using both the standard static EIT

https://clinicaltrials.gov/ct2/show/NCT02962505?term=NCT02962505\&rank $\underline{=1}$ 


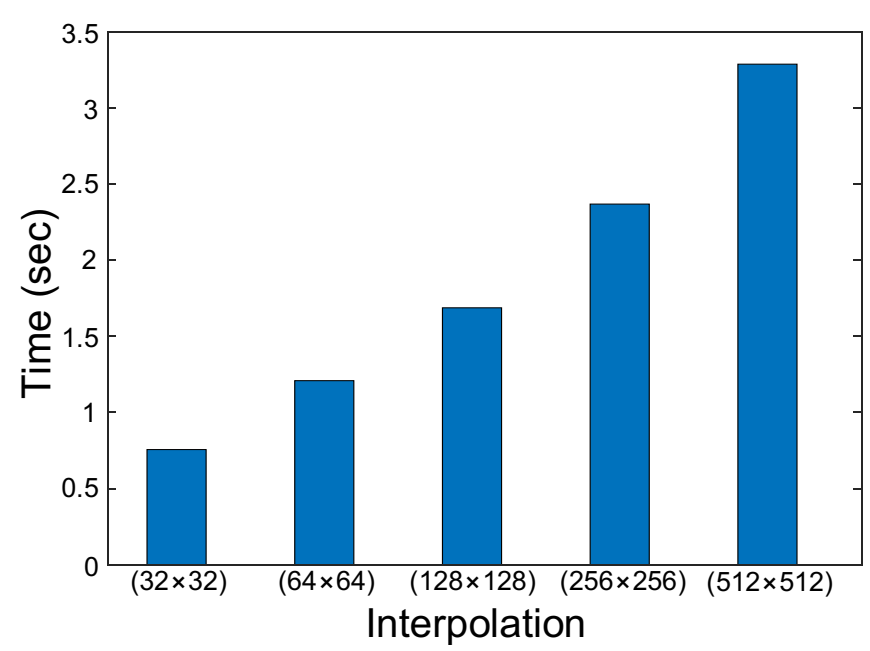

Fig. 16. Processing time to trace AT in an enhanced EIT image as a function of image size.

images $\left(F I_{E I T}\right)$ and the resolution enhanced EIT images with AT tracing $\left(F I_{A T}\right)$. The measured $F I_{E I T}$ and $F I_{A T}$ for pre and post inhalations are listed in Table A.1 in the Appendix.

The standard deviation $\sigma$ of the four lung quadrants (RA, RP, LA and LP) of the pre and post FIs for each subject provides a suitable metric for quantifying how uniformly (or nonuniformly) the air is exchanged over the quadrants, where:

$$
\sigma=\sqrt{\frac{1}{4} \sum_{j=\mathrm{RA}}^{\mathrm{RP}, \mathrm{LA}, \mathrm{LP}}\left(F I_{j}-\mu\right)^{2}}
$$

and $\mu=\frac{1}{4} \sum_{i=\mathrm{RA}}^{\mathrm{RP}, \mathrm{LA}, \mathrm{LP}} F I_{i}$. The mean standard deviation $\sigma_{m}$ for all the 10 subjects were: $\operatorname{pre}\left(F I_{E I T}\right) \sigma_{m}=0.6865 ; \operatorname{post}\left(F I_{E I T}\right)$ $\sigma_{m}=0.4266 ; \operatorname{pre}\left(F I_{A T}\right) \sigma_{m}=0.2170 ;$ and $\operatorname{post}\left(F I_{A T}\right) \sigma_{m}=$ 0.0767 .

For both $F I_{E I T}$ and $F I_{A T}$ the pre to post reduction of $\sigma_{m}$ shows evidence of lung function improvement as a result of clinical intervention. There is a relatively large difference between the pre and post $\sigma_{m}$ of $F I_{E I T}$ compared with $F I_{A T}$. An extra $\sigma_{m}$ component is associated with the overall accumulated error in $F I_{E I T}$ and will be added to the true $\sigma_{m}$ when determining the FIs. The larger difference is because the EIT image reflects on the cross-sectional plane and is not error-free. It becomes worse when impedance values in each quadrant are accumulated and fitted against the global impedance for FI derivation. The transition using $F I_{A T}$ is smoother (i.e. less deviation from the pre to post) and it shows more optimal air exchange in the quadrants due to clinical intervention. The AT tracing algorithm performs reverse processing embedding with more resolution than standard EIT images. It identifies the AT through decorrelation steps of the EIT plane and delivers an almost error-free AT matrix for the FI calculation.

\section{Timing Analysis}

A recognised technique to compare the computational complexity of algorithms is by measurement of their execution times, which are correlated with the number of arithmetic operations (i.e. additions or subtractions and multiplications or divisions). Fig. 16 shows the processing time of the AT algorithm with the standard and resized EIT images running on an Intel Dual Core $2.4 \mathrm{GHz}$ processor with $16 \mathrm{~GB}$ RAM. The processing time increases almost linearly with respect to the area of the image. This is because the size of the enhancement decomposition kernels in RQM [15] are adjusted based on the resized EIT image, and the AT tracing algorithm is optimized to perform multi-parallel recognition of the partial trees and ultimately forming the global AT. As the bicubic interpolation factor is increased, more intense processing is needed to not only enhance image patches, but also in dense rendering of the enhanced image to fully extract the partial trees and reach the full AT. The process time/detailed image trade off consideration applies when, for instance, monitoring and extracting correct branching layers as well as small variations in AT branching in some applications like template matching.

\section{CONCLUSION}

A method has been proposed for accurate estimation of recruitable alveolar collapse and hyperdistension from EIT images. The ANCC re-calibrates the FI in each quadrant during the respiration phase. To calculate the ANCC, the EIT images have been resized and enhanced to identify the effective paths corresponding to air circulation in the lungs. A regional enhancement method based on embedding of multi-scale projections of EIT images has been adopted. The resolution enhancement integrates multi-scale projection of the EIT image to identify hidden variations. Analyses have shown that the branching in the lungs follow the air flow field patterns which match the spatial orientations in the EIT image. The spatial orientations help with recognition of branching as well as branch segments linking nodes for tracking the AT. The computed information has been applied to the proposed tracing algorithm which automatically scored the probability of tree segments in EIT images without the need for user intervention. The proposed tracing algorithm can efficiently handle various configurations in partial branching and displays a reliable AT. Based on EIT images generated from resistive phantoms, the extracted AT modifies the FI which results in a correction modification of $65 \%$. The FI based on both the standard EIT and AT has been examined in a clinical setting for 10 subjects. They were subjected to inhalation of drugs under control of a clinician to improve the circulation of the air in the four quadrants of their lungs. The mean standard deviation of the four quadrants of the 10 subjects has been used as a measure of their degree of average uniformity of air distribution. The mean standard deviations before and after inhalation have indicated that the measured average improvement of air circulation is more accurate using the AT algorithm than the standard EIT algorithm.

\section{REFERENCES}

[1] B. Brown, "Electrical impedance tomography (EIT): A review," J. Med. Eng. Technol., vol. 27, no. 3, pp. 97-108, 2003.

[2] M. B. P. Amato, C. S. V. Barbas, D. M. Medeiros et al., "Effect of a protective-ventilation strategy on mortality in the acute respiratory distress syndrome," N. Engl. J. Med., vol. 338, no. 6, pp. 347-354, 1998.

[3] V. M. Ranieri, P. M. Suter, C. Tortorella et al., "Effect of mechanical ventilation on inflammatory mediators in patients with acute respiratory distress syndrome: a randomized controlled trial," J. American Medical Association, vol. 282, no. 1, pp. 54-61, 1999.

[4] F. B. Plotz, A. S. Slutsky, A. J. van Vught, and C. J. Heijnen, "Ventilatorinduced lung injury and multiple system organ failure: a critical review of 
facts and hypotheses," Intensive Care Med., vol. 30, no. 10, pp. 1865$1872,2004$.

[5] C.A. Grant, J.F. Fraser, K.R. Dunster, and A. Schibler "The assessment of regional lung mechanics with electrical impedance tomography: a pilot study during recruitment manoeuvres," Intensive Care Med., vol. 35, no. 1, pp. 166-170, 2009.

[6] D. Rooney, M. Friese, J.F. Fraser, K.R. Dunster, and A. Schibler, "Gravity-dependent ventilation distribution in rats measured with electrical impedance tomography," Physiol Meas., vol. 30, pp. 1075$1085,2009$.

[7] J. Hinz, A. Gehoff, O. Moerer, I. Frerichs, G. Hahn, G. Hellige, and M. Quintel "Regional filling characteristics of the lungs in mechanically ventilated patients with acute lung injury," Eur. J. Anaesthesiol., vol. 24, no. 5, pp. 414-424, 2007.

[8] M. van Heerde, K. Roubik, V. Kopelent, M.C.J. Kneyber, and D.G. Markhorst, "Spontaneous breathing during high-frequency oscillatory ventilation improves regional lung characteristics in experimental lung injury," Acta Anaesthesiol. Svand., vol. 54, no. 10, pp. 1248-1256, 2010.

[9] I. Frerichs, T. Dudykevych, J. Hinz, M. Bodenstein, G. Hahn, and G. Hellige, "Gravity effects on regional lung ventilation determined by functional EIT during parabolic flights," J. Appl. Physiol., vol. 91, no. 1, pp. 39-50, 2001.

[10] E. L. V. Costa, J. B. Borges, A. Melo, F. Suarez-Sipmann, C. Toufen, S. H. Bohm, and M. B. P. Amato, "Bedside estimation of recruitable alveolar collapse and hyperdistension by electrical impedance tomography," Intens. Care Med., vol. 35, pp. 1132-1137, 2009.

[11] R. Yerworth, and R. Bayford, "The effect of serial data collection on the accuracy of electrical impedance tomography images," Physiol. Meas., vol. 34, no. 6, pp. 659-669, 2013.

[12] J.-L. Starck, F. Murtagh, E. J. Candes, and D. L. Donoho, "Gray and color image contrast enhancement by the curvelet transform," IEEE Trans. Image Process., vol. 12, no. 6, pp. 706-717, Jun. 2003.

[13] J. Kim, J. K. Lee, and K. M. Lee, "Accurate image super-resolution using very deep convolutional networks,"' in Proc. IEEE Conf. Comput. Vis. Pattern Recognit. (CVPR), Jun. 2016, pp. 1646-1654.

[14] J. K. Kim, J. M. Park, K. S. Song, and H. W. Park, "Adaptive mammographic image enhancement using first derivative and local statistics," IEEE Trans. Med. Imag., vol. 16, no. 5, pp. 495_502, Oct. 1997.

[15] M. Zamani, R. Bayford, and A. Demosthenous, "Adaptive electrical impedance tomography resolution enhancement using statistically quantized projected image sub-bands," IEEE Access, vol. 8, pp. 99797$99805,2020$.

[16] W. T. Freeman and E. H. Adelson, "The design and use of steerable filters," IEEE Trans. Pattern Anal. Mach. Intell., vol. 13, no. 9, pp. 891906, 1991.

[17] K. Horsfield, G. Dart, D. E. Olson, G. F. Filley, G. Cumming, "Models of the human bronchial tree," J. Appl. Physiol., vol. 31, pp. 207-217, 1971.

[18] H. Kitaoka, R. Takaki and B. Suki, "A three-dimensional model of the human airway tree," J. Appl. Physiol., vol. 87, no. 6, pp. 2207-2217, Dec. 1999.

[19] M. H. Tawhai, P. Hunter, J. Tschirren, J. Reinhardt, G. McLennan and E. A. Hoffman, "CT-based geometry analysis and finite element models of the human and ovine bronchial tree," J. Appl. Physiol., vol. 97, no. 6, pp. 2310-2321, Dec. 2004.

[20] M. E. Martinez-Perez, A. D. Hughes, A. V. Stanton, S. A. Thom, N. Chapman, A. A. Bharath, and K. H. Parker, "Retinal vascular tree morphology: A semiautomatic quantification," IEEE Trans. Biomed. Eng., vol. 49, no. 8, pp. 912-917, Aug. 2002.

[21] M. Jridi et al., "A generalized algorithm and reconfigurable architecture for efficient and scalable orthogonal approximation of DCT," IEEE Trans. Circuits Syst. I, Reg. Papers, vol. 62, no. 2, pp. 449-457, Feb. 2015.

[22] A. R. Rao, A Taxonomy for Texture Description and Identification, New York: Springer-Verlag, 1990.

[23] N. Seifnaraghi et al., "Model selection based algorithm in neonatal chest EIT," IEEE Trans. Biomed Eng, Reg., vol. 68, no. 9, pp. 2752- 2763, Sep. 2021

[24] P. Lo, J. Sporring, H. Ashraf, J. J. H. Pedersen, and M. de Bruijne, "Vessel-guided airway tree segmentation: A voxel classification approach," Med. Image Anal., vol. 14, no. 4, pp. 527-538, Aug. 2010.

[25] A. Kiraly, W. Higgins, E. Hoffman, G. McLennan and J. Reinhardt, "3D human airway segmentation for virtual bronchoscopy," in Physiology and Function from Multidimensional Images, Proc. SPIE, vol. 4683, 2002.

[26] P. J. Reynisson, M. Scali, E. Smistad, E. F. Hofstad, H. O. Leira, F. Lindseth, T. A. Nagelhus Hernes, T. Amundsen, H. Sorger, and T.
Table A.1

FI Values $F I_{E I T}$ and $F I_{A T}$ for the Four Lung Quadrants of 10 Subjects Before (Pre) and After (Post) Inhalation.

\begin{tabular}{|c|c|c|c|c|c|}
\hline \multirow{3}{*}{ Subjects $^{\mathrm{a}}$} & & \multicolumn{4}{|c|}{ Filling index $(\mathrm{FI})$} \\
\hline & & \multicolumn{2}{|c|}{$F I_{E I T}$} & \multicolumn{2}{|c|}{$F I_{A T}$} \\
\hline & & Pre & Post & Pre & Post \\
\hline \multirow{4}{*}{$\begin{array}{c}\text { Subject } 1 \\
\text { HFNC }^{\mathrm{b}} \\
\text { Ipratropium }\end{array}$} & $\mathrm{RP}$ & 1.597 & 0.5577 & 0.2660 & 0.0247 \\
\hline & RA & 0.00728 & 0.0515 & 0.1586 & 0.0181 \\
\hline & LP & -0.6071 & -0.6071 & -0.0465 & -0.0466 \\
\hline & LA & -1.0348 & -0.0021 & -0.0674 & -0.0456 \\
\hline \multirow{4}{*}{$\begin{array}{c}\text { Subject } 2 \\
\text { HFNC } \\
\text { Salbutamol }\end{array}$} & $\mathrm{RP}$ & 2.7200 & -0.5420 & 0.1507 & -0.1028 \\
\hline & RA & -0.8200 & 1.2670 & -0.5250 & 0.2348 \\
\hline & LP & 0.6810 & -1.8160 & 0.6225 & -0.0818 \\
\hline & LA & -1.2200 & 1.0910 & -0.2400 & 0.1125 \\
\hline \multirow{4}{*}{$\begin{array}{c}\text { Subject } 3 \\
\text { HFNC } \\
\text { Salbutamol }\end{array}$} & RP & 0.0640 & 0.2500 & 0.4332 & 0.2042 \\
\hline & RA & -1.6130 & -0.5400 & -0.3049 & -0.0789 \\
\hline & LP & 0.7600 & 0.3800 & 0.2641 & 0.1216 \\
\hline & LA & 0.7800 & -0.2700 & -0.5472 & -0.2527 \\
\hline \multirow{4}{*}{$\begin{array}{c}\text { Subject } 4 \\
\text { Invasive }^{\mathrm{c}} \\
\text { Salbutamol }\end{array}$} & $\mathrm{RP}$ & 0.3670 & 0.3240 & 0.0152 & 0.0323 \\
\hline & RA & 0.0930 & 0.0450 & 0.0864 & 0.0542 \\
\hline & LP & -0.0550 & -0.4720 & -0.0732 & -0.0570 \\
\hline & LA & -0.4050 & -0.1960 & -0.0275 & -0.0123 \\
\hline \multirow{4}{*}{$\begin{array}{c}\text { Subject } 5 \\
\text { Invasive } \\
\text { Salbutamol }\end{array}$} & $\mathrm{RP}$ & 0.3610 & 0.1250 & 0.1118 & 0.0338 \\
\hline & RA & -0.2390 & -0.1960 & -0.0988 & -0.0188 \\
\hline & LP & 0.4780 & 0.3800 & 0.0286 & 0.0059 \\
\hline & LA & -0.1710 & -0.3095 & -0.1229 & -0.1280 \\
\hline \multirow{4}{*}{$\begin{array}{c}\text { Subject } 6 \\
\text { Invasive } \\
\text { Salbutamol }\end{array}$} & $\mathrm{RP}$ & 0.2899 & 0.0360 & 0.0117 & 0.0241 \\
\hline & RA & -0.0328 & -0.1146 & -0.0306 & -0.0074 \\
\hline & LP & 0.1240 & -0.0885 & 0.8685 & 0.0071 \\
\hline & LA & -0.3651 & 0.1660 & -0.0191 & -0.0402 \\
\hline \multirow{4}{*}{$\begin{array}{c}\text { Subject } 7 \\
\text { Invasive } \\
\text { Ipratropium }\end{array}$} & $\mathrm{RP}$ & 0.3530 & 0.1560 & 0.0253 & 0.0099 \\
\hline & RA & -0.1503 & -0.1240 & -0.0550 & -0.0187 \\
\hline & LP & 0.1176 & 0.1195 & 0.0467 & 0.0154 \\
\hline & LA & -0.3197 & -0.1515 & -0.0198 & -0.0336 \\
\hline \multirow{4}{*}{$\begin{array}{c}\text { Subject } 8 \\
\text { HFNC } \\
\text { Ipratropium }\end{array}$} & RP & 0.4660 & 0.4270 & 0.0071 & 0.0106 \\
\hline & RA & 0.0819 & 0.0252 & 0.1050 & 0.0423 \\
\hline & LP & -0.0502 & -0.0165 & -0.0865 & -0.0572 \\
\hline & LA & -0.4680 & -0.2860 & -0.0198 & -0.0059 \\
\hline \multirow{4}{*}{$\begin{array}{c}\text { Subject } 9 \\
\text { Invasive } \\
\text { Salbutamol }\end{array}$} & $\mathrm{RP}$ & 0.4008 & 0.1529 & 0.0975 & 0.0056 \\
\hline & RA & -0.1133 & -0.0786 & -0.0990 & -0.0705 \\
\hline & LP & 0.2659 & 0.1339 & 0.0585 & 0.0315 \\
\hline & LA & -0.4108 & -0.3653 & -0.0574 & -0.0337 \\
\hline \multirow{4}{*}{$\begin{array}{c}\text { Subject } 10 \\
\text { HFNC } \\
\text { Salbutamol }\end{array}$} & $\mathrm{RP}$ & 1.0910 & 0.3540 & 0.2082 & 0.0473 \\
\hline & RA & -0.5290 & -0.1123 & -0.2044 & -0.1674 \\
\hline & LP & 0.1151 & 0.2390 & 0.2022 & 0.0503 \\
\hline & LA & -0.6154 & -0.5811 & -0.0980 & -0.0665 \\
\hline \multicolumn{2}{|l|}{$\begin{array}{l}\text { Mean standard } \\
\text { deviation }\left(\sigma_{m}\right)\end{array}$} & 0.6865 & 0.4266 & 0.21704 & 0.0767 \\
\hline
\end{tabular}

${ }^{a}$ Subjects were 10-28 months old and weighing 7-13 kg.

${ }^{\mathrm{b}}$ HFNC (high flow nasal cannula).

${ }^{\mathrm{c}}$ Invasive ventilation.

Langø, "Airway segmentation and centerline extraction from thoracic CT-comparison of a new method to state of the art commercialized methods," PLoS ONE, vol. 10, no. 12, Dec. 2015, Art. no. e0144282.

[27] Sentec AG, Switzerland. "Sentec EIT Pioneer Set" product information. Accessed: June 21, 2021. Available: http://www.swisstom.com/wpcontent/uploads/SenTec-EITPioneerSet-Product-Information-1ST500102Rev008-web.pdf

[28] A. Adler et al, "GREIT: A unified approach to 2D linear EIT reconstruction of lung images," Physiol. Meas., vol. 30, pp. S35-S55, 2009.

[29] A. Hauptmann et al., "Open 2D electrical impedance tomography data archive," arXiv preprint arXiv: 1704.01178, 2017.

[30] M. Cousineau, A. Adler and A. E. Hartinger, "A resistive phantom for the assessing the performance of EIT systems," IEEE Trans. Biomed. Eng., vol. 57, no, 9, pp. 2257-2266, 2010. 


\section{APPENDIX}

Table A.1 lists the calculated FI values $F I_{E I T}$ and $F I_{A T}$ for pre and post inhalations for the 10 subjects. A positive polynomial coefficient shows possible regional collapse or quadrant lagging and a negative polynomial coefficient shows the possible hyperdistention or leading of a quadrant to the whole lung. Also, the calculated $\sigma_{m}$ for all the subjects is listed in the last row of Table A.1. 